\title{
Clinical pharmacology strategies in supporting drug development and approval of antibody-drug conjugates in oncology
}

\author{
Stephanie N. Liu ${ }^{1} \cdot$ Chunze $_{\mathrm{Li}^{1}}{ }^{\mathbb{D}}$
}

Received: 14 September 2020 / Accepted: 18 February 2021 / Published online: 1 April 2021

(c) The Author(s) 2021

\begin{abstract}
Antibody-drug conjugates (ADCs) are important molecular entities in the treatment of cancer. These conjugates combine the target specificity of monoclonal antibodies with the potent anti-cancer activity of small-molecule therapeutics. The complex structure of ADCs poses unique challenges to characterize the drug's pharmacokinetics (PKs) and pharmacodynamics (PDs) since it requires a quantitative understanding of the PK and PD properties of multiple different molecular species (e.g., ADC conjugate, total antibody and unconjugated cytotoxic drug). As a result, clinical pharmacology strategy of an ADC is rather unique and dependent on the linker/cytotoxic drug technology, heterogeneity of the ADC, PK and safety/efficacy profile of the specific ADC in clinical development. In this review, we summarize the clinical pharmacology strategies in supporting development and approval of ADCs using the approved ADCs as specific examples to illustrate the customized approach to clinical pharmacology assessments in their clinical development.
\end{abstract}

Keywords Antibody-drug conjugate $\cdot$ Clinical pharmacology $\cdot$ Population pharmacokinetics $\cdot$ Exposure-response analysis $\cdot$ Specific population $\cdot$ Drug interaction $\cdot$ QTc prolongation

\section{Introduction}

Antibody drug conjugates (ADCs) are an emerging class of anti-cancer therapeutic agents that combine the antigen targeting specificity and favorable pharmacokinetic properties of monoclonal antibodies (mAbs) with the cytotoxic potential of small-molecule chemotherapeutics [1]. ADCs typically consist of three components, namely a mAb to determine which cells to be targeted, a cytotoxic drug to determine the mechanism of action by which cells are killed, and a chemical linker that attaches these two components together to determine how the drug is released. The $\mathrm{mAb}$ component of an ADC enables the ADC to specifically bind to targeted cell surface antigens overexpressed on the tumor cells. Upon binding, the ADCs are internalized and trafficked to lysosomes, from which the cytotoxic drug is released within the cell, thus resulting in the cell death. The use of targeted delivery of highly potent cytotoxic drugs is

Chunze Li

li.chunze@gene.com

1 Department of Clinical Pharmacology, Genentech, Inc, 1 DNA Way, South San Francisco, CA 94080, USA designed to enhance the antitumor effects of the molecule while minimizing the toxicity in the normal tissues.

As of January 2020, nine ADCs have received US Food and Drug Administration (FDA) approval [2]. The first of these, (1) gemtuzumab ozogamicin (Mylotarg $\AA$; an antiCD33 mAb linked to calicheamicin), for the treatment of acute myelogenous leukemia (AML) was approved in 2000 under the FDA accelerated-approval process [3]. In 2010, this agent was voluntarily withdrawn from the market due to confirmatory trials failing to demonstrate clinical benefit and safety concerns [3]. Gemtuzumab ozogamicin was re-approved in 2018 at a sub-fractionated dose of 3-6 mg/ $\mathrm{m}^{2}$ (compared to $9 \mathrm{mg} / \mathrm{m}^{2}$ at first approval) [4]. Since gemtuzumab ozogamicin's initial market approval, seven more ADCs were FDA approved: (2) brentuximab vedotin (Adcetris ${ }^{\circledR}$; an anti-CD30 mAb and monomethyl auristatin E [MMAE] conjugate) for the treatment of Hodgkin lymphoma and systemic anaplastic large-cell lymphoma, (3) trastuzumab emtansine (T-DM1, Kadcyla ${ }^{\circledR}$; an anti-human epidermal growth factor receptor 2 (HER2) $\mathrm{mAb}$ and DM1 [a derivative of maytansine] conjugate) for the treatment of HER2 + metastatic breast cancer (mBC), (4) inotuzumab ozogamicin (Besponsa ${ }^{\circledR}$, an anti-CD22 mAb and calicheamicin conjugate) for the treatment of adults with relapsed or 
refractory B-cell precursor acute lymphoblastic leukemia (ALL), (5) polatuzumab vedotin (Polivy ${ }^{\circledR}$, an anti-CD79b $\mathrm{mAb}$ and MMAE conjugate) for the treatment of relapsed or refractory diffuse large B-cell lymphoma (DLBCL), (6) enfortumab vedotin $\left(\right.$ Padcev ${ }^{\circledR}$, an anti-Nectin $4 \mathrm{mAb}$ and MMAE conjugate) for the treatment of locally advanced or metastatic urothelial cancer, (7) trastuzumab deruxtecan (Enhertu ${ }^{\circledR}$, an anti-HER2 $\mathrm{mAb}$ and exatecan derivative conjugate) for the treatment of HER2 $+\mathrm{mBC}$, and (8) sacituzumab govitegcan (Trodelvy ${ }^{\circledR}$, an anti-Trop-2 mAb and $\mathrm{SN}-38$ conjugate) for the treatment of metastatic triplenegative breast cancer [5-11]. In August 2020, the 9th ADC, namely belantamab mafodotin-blmf (Blenrep ${ }^{\circledR}$, an antiBCMA mAb and MMAF conjugate) achieved accelerated approval from FDA for the treatment relapsed and refractory multiple myeloma [12].

These ADCs prove that the therapeutic window of otherwise intolerable cytotoxic drugs can be improved to a therapeutically beneficial level by conjugating it to an antibody. Despite the great success of ADCs, it is worth noting that the therapeutic window for ADCs remains relatively narrow with the maximum tolerated dose (MTD) often reached before ADCs achieve the maximal efficacious dose [13]. As a result, numerous innovative approaches (e.g., site-specific conjugation or novel payloads) have been implemented to further improve the therapeutic window, resulting in the "next-generation" ADCs, many of which are currently tested in clinical development.

The current understanding of the mechanism at which ADCs are cleared is through two major pathways: proteolytic degradation and deconjugation $[14,15]$. ADC clearance through proteolytic degradation is driven primarily by catabolism mediated by target-specific or nonspecific cellular uptake followed by lysosomal degradation, similar to mAbs. Deconjugation clearance is usually mediated by enzymatic or chemical cleavage (e.g., maleimide exchange) of the linker leading to the release of the cytotoxic drug from the ADC [16]. Once released from the ADC, the cytotoxic drug may be further metabolized, transported, and eliminated via traditional mechanisms applicable to small molecules (see DDI section). Alternatively, ADC catabolism and deconjugation in vivo leads to the formation of multiple different molecular species (e.g., ADC species with different drug antibody ratios [DAR]) and payload-containing catabolites) [17]. The bioanalytical strategy for ADCs thus requires defining the specific analytes of relevance to clinical pharmacology. Although multiple analytes may be quantified following the dosing of an ADC, the clinical importance of the multi-analyte bioanalytical data in context of safety and efficacy remains to be established. With numerous ADCs in clinical development, streamlining the bioanalytical and clinical pharmacology strategy is critical.

The clinical pharmacology assessments for ADCs to address scientific and regulatory concerns are summarized in Fig. 1. The clinical pharmacology of an ADC incorporates elements of small molecule and mAb (large molecule) development strategies. The scope of work is usually unique and dependent on the linker/cytotoxic drug technology, heterogeneity of the ADC, pharmacokinetics (PK) and safety/efficacy profile of the specific ADC in clinical development. Given the structural complexity, multiple analytes were measured across Phase I, Phase II and Phase III clinical studies to characterize the PK of an ADC, including, but not limited to, conjugate, total antibody and unconjugated payload.

\begin{tabular}{|c|c|c|}
\hline PRECLINICAL & CLINICAL & \\
\hline$>\begin{array}{c}\text { Preclinical } \\
\text { Development }\end{array}$ & $\begin{array}{c}\text { Confirmatory } \\
\text { Phase III }\end{array}$ & $\begin{array}{l}\text { Approval/ } \\
\text { Post- } \\
\text { Marketing }\end{array}$ \\
\hline $\begin{array}{l}\text { - In vitro and in vivo ADME } \\
\text { characterization } \\
\text { - PK characterization: multiple } \\
\text { analytes (semi-mechanistic } \\
\text { multiple analyte PK models) } \\
\text { - Bioanalytical strategy } \\
\text { - Human PK prediction } \\
\text { - Human dose projection: FIH } \\
\text { starting dose and projected } \\
\text { efficacious dose } \\
\text { (Translational PKPD } \\
\text { modeling) }\end{array}$ & $\begin{array}{l}\text { - ADCs PK characterization: multiple analytes (NCA, and } \\
\text { multiple-analyte popPK) } \\
\text { - Dose and schedule selection (exposure-response analysis, } \\
\text { PKVPD) } \\
\text { - Effect of intrinsic factors: BW, race, age, liver/renal } \\
\text { impairment (PopPK, PBPK, dedicated clinical study) } \\
\text { - Effect of extrinsic factors: Drug drug interaction, material } \\
\text { comparability (PopPK, PBPK, dedicated clinical study) } \\
\text { - Concentration-QTc assessment (integrated to existing } \\
\text { clinical Phase I/II study, dedicated clinical study) } \\
\text { - Immunogenicity and its impact on safety and efficacy } \\
\text { - Combination strategy (QSP, PKPD) }\end{array}$ & $\begin{array}{l}\text { - Clinical dose } \\
\text { justification } \\
\text { - Clinical } \\
\text { pharmacology } \\
\text { characterization } \\
\text { - Decision } \\
\text { making } \\
\text { - Drug label }\end{array}$ \\
\hline
\end{tabular}

Fig. 1 Clinical pharmacology considerations in the stages of ADC drug development 
Intrinsic (e.g., body weight, organ dysfunction) and extrinsic factors (e.g., concomitant medications) likely to impact the PK of an ADC were assessed using diverse approaches (Fig. 1). Relationships between exposure to ADC conjugate (and other relevant analytes) and efficacy/safety were assessed in support of the clinical dosing regimen selection using quantitative approaches. This review summarized the unique clinical pharmacology consideration in supporting development and approval of ADCs (Fig. 1). The seven approved ADCs are used as specific examples to illustrate the customized approach to clinical pharmacology assessments in their clinical development. Sacituzumab govitecan and belantamab mafodotin-blmf were not included in the summary due to limited clinical pharmacology information available at the time of the review.

\section{Bioanalytical consideration}

ADCs incorporate both large- and small-molecule characteristics and are usually present as a heterogeneous mixture of the species differing not only in the number of cytotoxic drugs attached to the antibody, but also in the protein conjugation sites of drug linkage [18]. Furthermore, biotransformations in vivo can lead to additional changes in DARs resulting in dynamically changing mixtures. As a result, unlike mAbs, the heterogeneity of ADCs in vivo makes it critical to measure multiple analytes in clinical trials [17, 19]. These analytes may include, but are not limited to, the following: ADC conjugate (measured as conjugated antibody or conjugated payload), total antibody (TAb, conjugated and unconjugated antibody), unconjugated antibody and unconjugated (free) payload. Conjugated antibody and conjugated payload are the two alternative ways to quantify the ADC conjugate [20]. From the perspective of the antibody, the ADC conjugate can be measured as "conjugated antibody", namely the concentration of antibody molecules with one or more cytotoxic drugs attached. This bioanalytical method is used to measure serum concentrations of ADC conjugate for brentuximab vedotin, inotuzumab ozogamicin, T-DM1, enfortumab vedotin, and trastuzumab deruxtecan [21-25]. Alternatively, from the perspective of the payload, the ADC conjugate can be measured as "conjugated drug", namely as the total concentration of cytotoxic drug that is conjugated to the antibody. Currently, only ADCs with cleavable linker are amenable to the conjugated drug assay. This bioanalytical method is used to measure polatuzumab vedotin given that not all the DAR species can be measured accurately in the conjugated-antibody ELISA assay [26]. In comparison, gemtuzumab ozogamicin does not measure ADC conjugate. Instead, gemtuzumab ozogamicin measured $\mathrm{TAb}$ and unconjugated calicheamicin [27, 28] likely due to the availability of bioanalytical techniques at the time of development.

Although multiple analytes may be quantified following the dosing of an ADC, the bioanalytical strategy for ADCs requires defining the specific analytes of relevance to clinical pharmacology in the context of safety and efficacy. Most commonly, three analytes, namely ADC conjugate, TAb and unconjugated cytotoxic drug are measured in preclinical and clinical studies to characterize the PK properties of an ADC [17, 19].

\section{Population PK modeling}

Population PK modeling is an important approach to characterize the ADC PK properties and assess the effect of intrinsic and extrinsic factors on ADC PK, and thus guide dose recommendations in specific populations (e.g., geriatric patients or patients with organ dysfunction). Given multiple analytes were measured for an ADC during its clinical development, one of the unique features of population PK for an ADC is that more than one analyte is often included in the population $\mathrm{PK}$ model development. ADC conjugate, the main analyte of interest per mechanism of action of ADCs, is the most common analyte included in the population PK model. Additionally, given the high potency of cytotoxic drugs, the potential contribution of unconjugated drug to safety could not be ruled out. Exposure-safety analysis with unconjugated cytotoxic drug has been conducted for the four out of the seven approved ADCs (see exposure-response section). As a result, unconjugated drug analyte is often included in the population PK model in addition to ADC conjugate to understand the $\mathrm{PK}$ characteristics of unconjugated drugs after ADC dosing and generate exposure metrics for exposure-response analysis.

As shown in Table 1, five out of the seven approved ADCs include the two analytes in their population PK models. Integrated two-analyte models (i.e., ADC conjugateunconjugated payload models) were developed for brentuximab vedotin, polatuzumab vedotin, enfortumab vedotin and trastuzumab deruxtecan, while for gemtuzumab ozogamicin population PK model for TAb and unconjugated payload was developed separately and ADC conjugate analyte was not measured clinically [28]. Typically, the ADC conjugate is dosed in the linear range based on the findings of the phase 1 dose escalation study. The population PK model structures for ADC conjugate are usually characterized by a 2- or 3compartment model with a mixture of linear and non-linear elimination pathways. Notably, three out of the seven ADCs have non-linear time-dependent clearance and all of them target hematological malignancy (Table 1). The ADC linear clearance $(\mathrm{CL}=1.6-2.5 \mathrm{~L} / \mathrm{day})$ and central volume of distribution $(\mathrm{Vc}=6.4-6.7 \mathrm{~L})$ are similar for brentuximab vedotin 


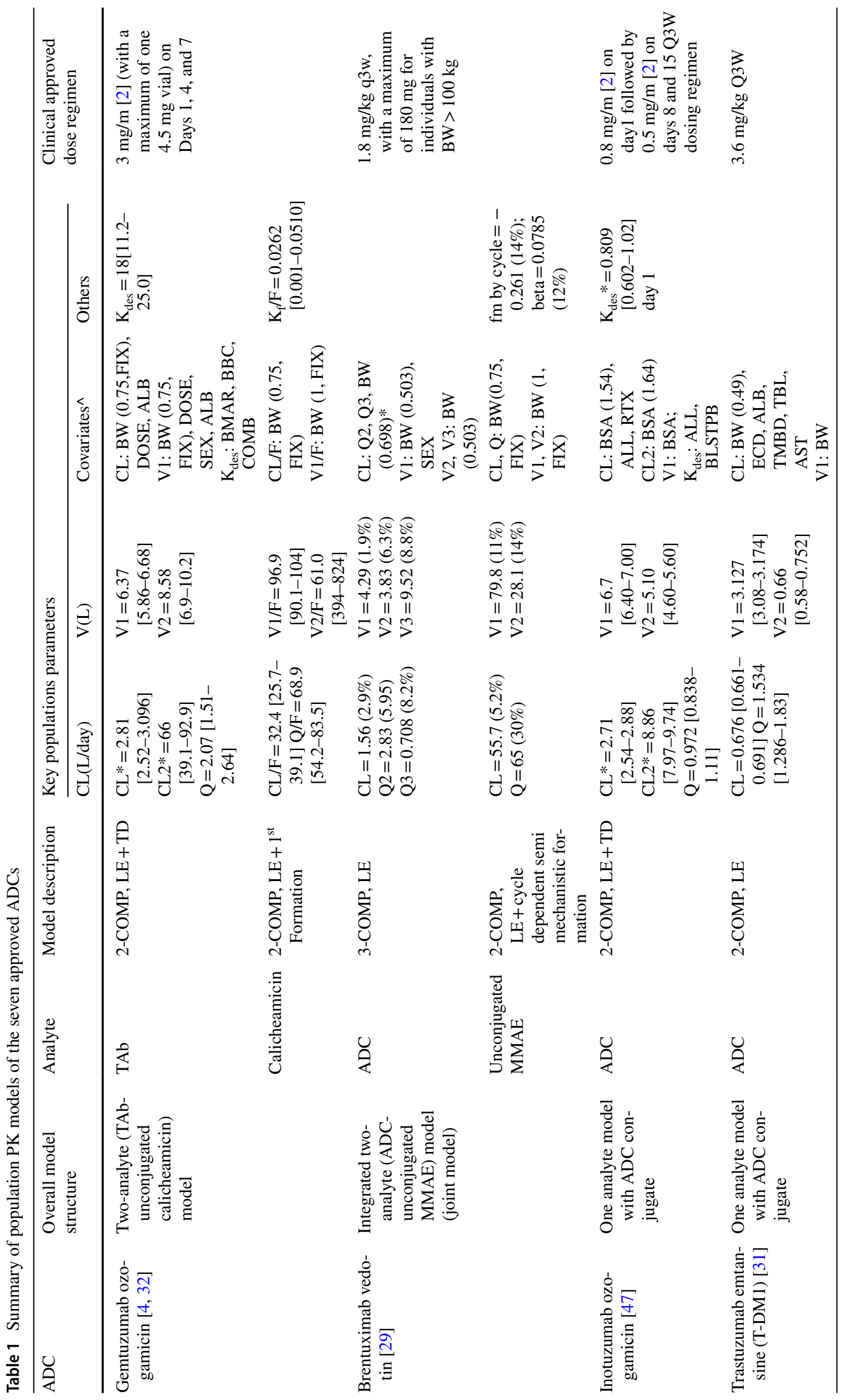




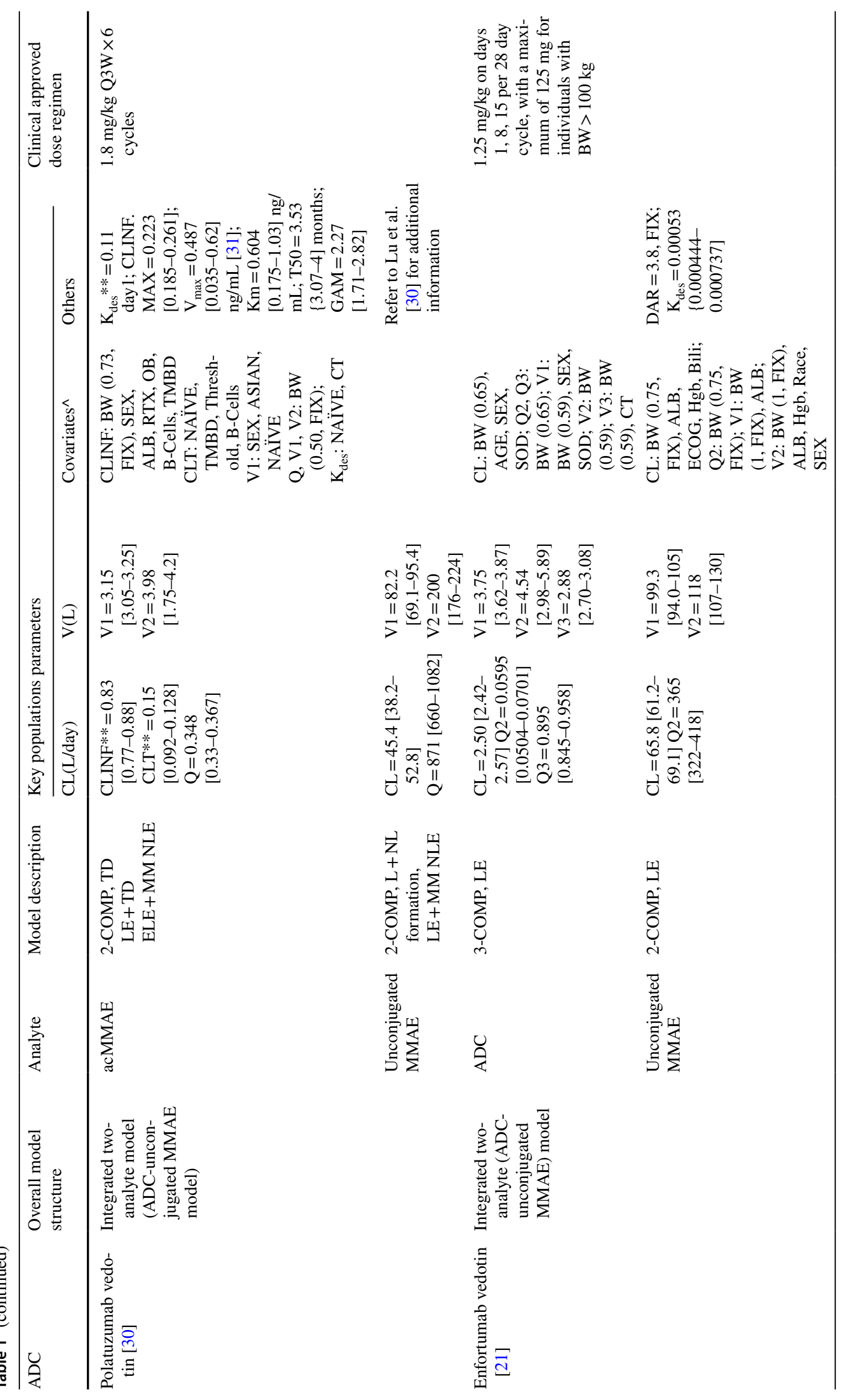




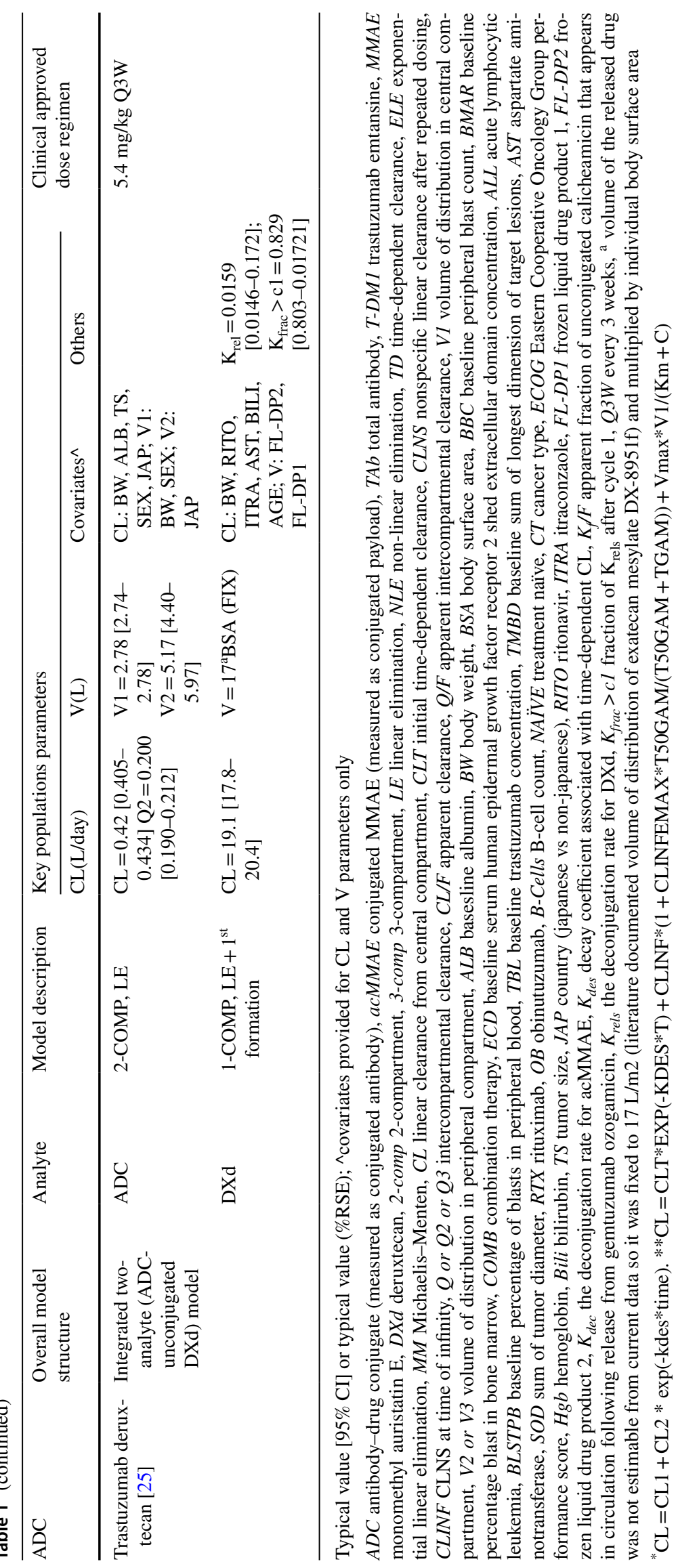


and enfortumab vedotin, the MMAE-containing ADCs that share the same cytotoxic drug and linker but against different targets [21, 29]. Polatuzumab vedotin is not included in the comparison due to apparent non-linear and time-dependent PK [30]. Conversely, T-DM1 and trastuzumab deruxtecan, both of which share the same mAb but with different cytotoxic drugs and linkers, exhibited linear PK with similar CL (0.4-0.7 L/day) and central volume of distribution ( 3 L) at the clinical approved dose [23, 31]. As expected for small molecules, the unconjugated payloads released from ADCs exhibit faster apparent clearances (>19 L/day) from circulation with larger apparent central volume of distribution $(>80 \mathrm{~L}$ ) into extravascular tissues compared to the ADC or TAb. For MMAE-containing ADCs, the MMAE apparent clearance and apparent central volume of distribution is 45-66 L/day and 80-99 L, respectively. The calicheamicin analyte was not characterized in the population PK model for inotuzumab ozogamicin so PK parameters for the payload were not available for comparison, but for gemtuzumab ozogamicin, unconjugated calicheamicin CL/F was $32 \mathrm{~L} /$ day and V1/F was $97 \mathrm{~L}$ [32]. For trastuzumab deruxtecan, the unconjugated DXd clearance $(\mathrm{CL}=19 \mathrm{~L} /$ day $)$ was the lowest for the payloads and the central volume was not estimable with data collected so it was fixed to nonclinical data in the population PK model [25].

The covariate effects from body weight (BW) or body surface area (BSA) is consistently identified as one of significant covariates on key PK parameters (i.e., CL and/or $\mathrm{Vc}$ ) in the final popPK models for all the approved ADCs. The exponential of BW effect on CL ranged from 0.49 to 0.75 , thus supporting the BW- and BSA- based dosing strategy for ADCs. It was worth noting that BW and BSA were highly correlated, of these two covariates, BW is usually preferred to be included in the model as it is the simpler measure to obtain. Six out of the seven approved ADCs identified BW as a significant covariate in their population PK model except inotuzumab ozogamicin which included BSA (Table 1). Among the seven approved ADCs, five of them utilized BW-based dosing regimen with the two calicheamicin-containing ADCs using BSA-based dosing. Since these agents have relatively narrow therapeutic windows, some of the ADCs (i.e., brentuximab vedotin, enfortumab vedotin) adopted dose capping strategy to further reduce inter-individual variability for the ADC exposure and thus potentially improve the ADC's safety profiles, particularly for patients with higher BW (i.e., BW $>100 \mathrm{~kg}$ ) that would achieve higher drug exposure from a weight-based dosing regimen $[21,22]$. Notably, no significant PK differences based on age was observed. Some differences in PK parameters with gender was observed, but post-hoc analyses showed it did not have any clinical meaningful effect on ADC exposures and thus did not warrant dosing adjustment based on gender. The impact of extrinsic and intrinsic factors on ADC PK has been discussed previously [33, 34]. Consistent with other biotherapeutics, baseline albumin and disease factors (e.g., tumor burden) were often identified as significant covariates for ADC clearance, however, the magnitude of the effect of these significant covariates on ADC exposure is minimal compared with overall PK variability and therefore the BWand BSA- based dose without further adjustment for other factors is considered appropriate for ADCs.

\section{Organ dysfunction studies}

The general concept that hepatic impairment may not affect therapeutic proteins $\mathrm{PK}$, including $\mathrm{mAbs}$ and ADCs, is being challenged with emerging evidence [34]. Recent publication by Sun et al. showed that of $20 \mathrm{mAbs}$ and 4 ADCs with hepatic impairment data, a decrease in exposure of $1 \mathrm{mAb}$ and 2 ADCs were observed in patients with hepatic impairment. Although the mechanism is unknown, Sun et al. proposes worsening of disease associated with hepatic impairment may increase the elimination of therapeutic proteins through increased competition of FcRn binding with other soluble proteins (i.e. albumin) and target mediated drug disposition. In addition, the liver and kidneys play an important role in elimination of the small-molecule component of an ADC, namely cytotoxic drug once it gets released from the ADC. As a result, impairment of the functions of these organs may result in alteration of $\mathrm{ADC}$ and/or cytotoxic drug clearance, leading to exposure changes, which may in turn impact the safety and efficacy. This is especially important given ADCs generally have a relatively narrow therapeutic index. Therefore, assessment of the impact of organ dysfunction on the disposition of ADCs to inform appropriate dosing in these patients is an important component of clinical pharmacology strategy for these molecules.

Table 2 summarizes the impact of liver and kidney function on ADCs PK and their corresponding dosing recommendation for the seven approved ADCs. It is noted that two alternative approaches were used to characterize the impact of organ dysfunction on ADC PK across the seven approved ADCs (1) a dedicated organ dysfunction clinical study or (2) model-based approach using patients with organ dysfunction across clinical studies to determine the effects of clinical PK. As shown in Table 2, three out of seven ADCs conducted dedicated hepatic and/or renal impairment clinical studies: brentuximab vedotin, T-DM1, and enfortumab vedotin. While for the remainder of ADCs, modeling and simulation through popPK has been utilized to assess the organ dysfunction subpopulation across clinical studies. The current ADC model-based approach requires that existing clinical studies allow enrollment of patients with organ impairment.

ADC conjugate exposure was generally comparable between patients with hepatic impairment and normal 


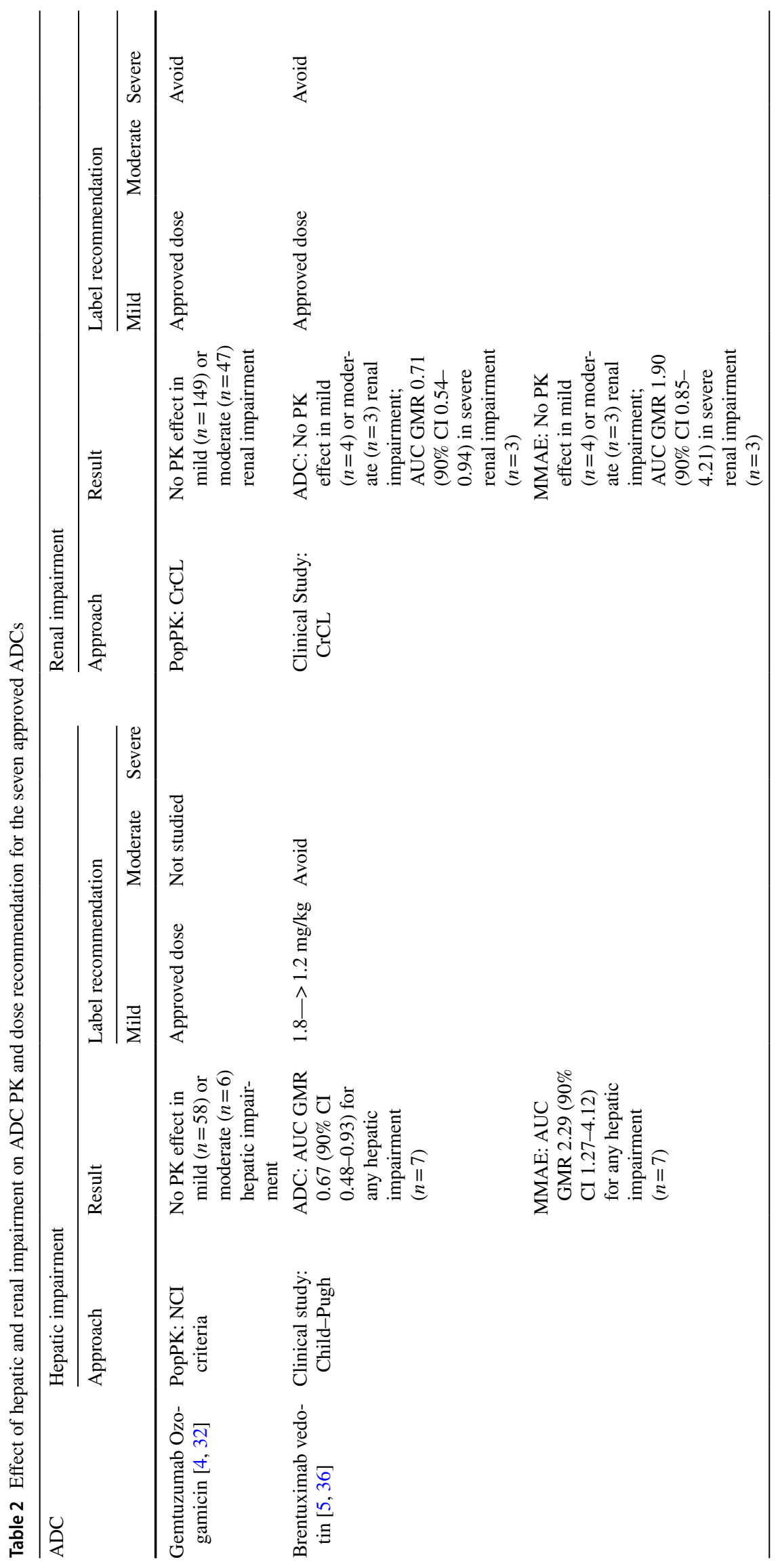




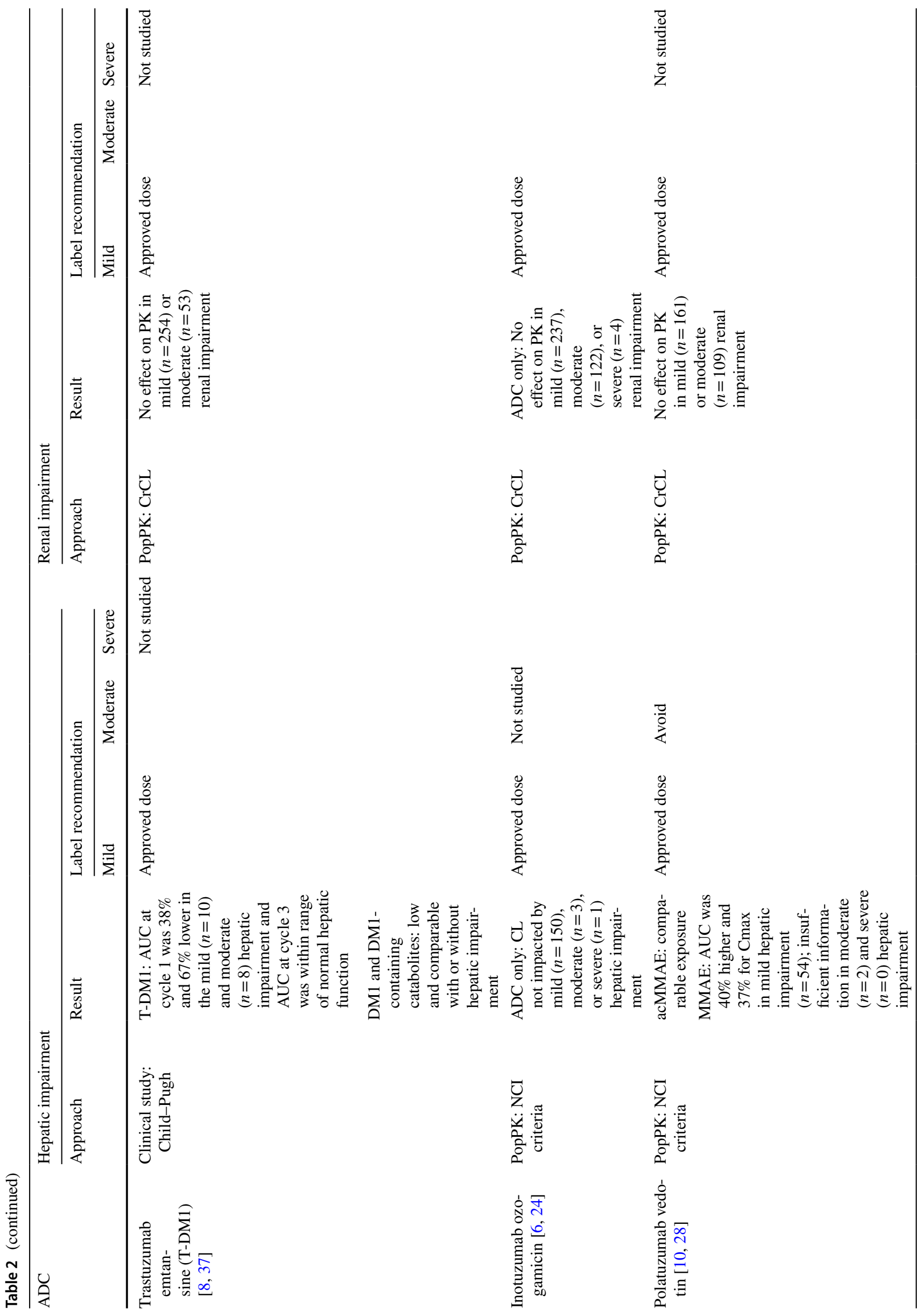




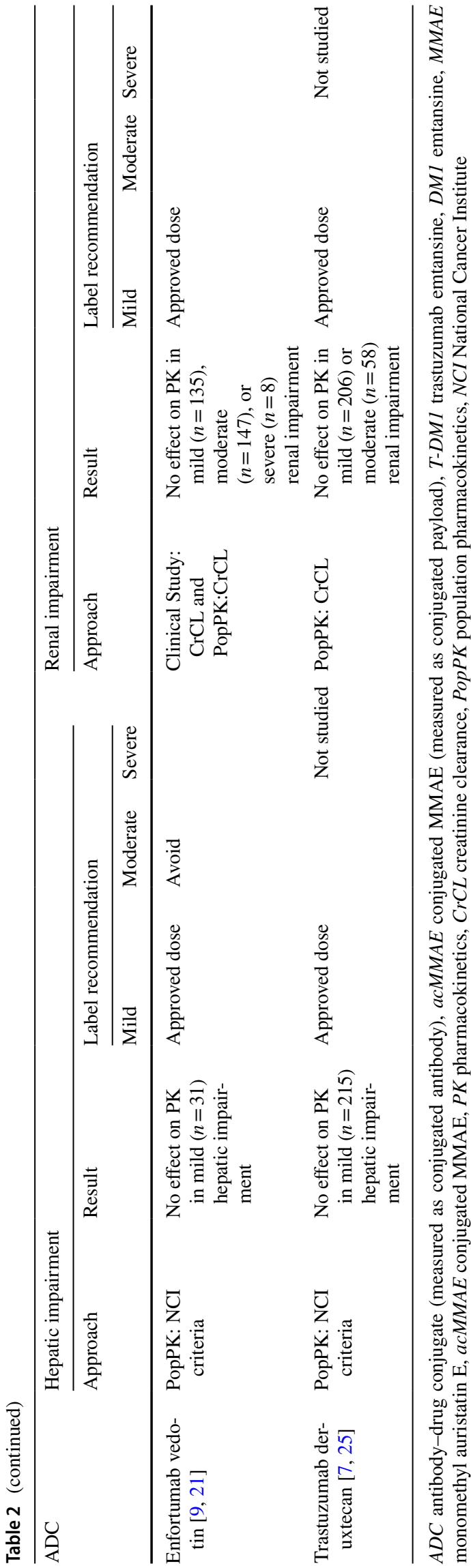

hepatic function for most of the approved ADCs, except for brentuximab vedotin and T-DM1 (Table 2). For brentuximab vedotin, ADC conjugate exposure (i.e., AUC) decreased by $35 \%$ in lymphoma patients with moderate hepatic impairment, and there was only one patient each with mild or severe hepatic impairment [35]. For T-DM1, AUC of T-DM1 conjugate at Cycle 1 in patients with mild and moderate hepatic impairment were approximately $38 \%$ and $67 \%$ lower than that of patients with normal hepatic function, respectively [36]. Interestingly, the exposure difference was less apparent after repeated dosing with T-DM1 AUC at Cycle 3 in patients with mild and moderate hepatic impairment largely comparable to the patients with normal hepatic function. There was no apparent effect of hepatic impairment on the cytotoxic drug exposure except for brentuximab vedotin (unconjugated MMAE AUC GMR 2.29 for any hepatic impairment vs normal hepatic function) and polatuzumab vedotin (unconjugated MMAE AUC GMR 1.40 for mild hepatic impairment vs normal hepatic function) $[26,36]$. The fact that the exposure of unconjugated MMAE was increased by two-to-threefold in moderate hepatic impaired patients resulted in label recommendation for brentuximab vedotin to avoid use in patients with moderate to severe hepatic impairment [5]. The comparable unconjugated DM1 exposure and transient change of T-DM1 conjugate exposure in mild or moderate hepatic impairment lead to label recommendation of no adjustments of the dose of T-DM1 in these patients [8, 37]. Although an increase in unconjugated MMAE exposure for polatuzumab vedotin was observed, based on the exposure-safety relationship established across clinical studies, the increased unconjugated MMAE exposure was not clinically relevant and no adjustment in the starting dose is required for polatuzumab vedotin in patients with mild hepatic impairment [26].

For patients with renal impairment, ADC conjugate and cytotoxic drug PK are comparable for most of the approved ADCs, except for brentuximab vedotin in patients with severe renal impairment (ADC AUC GMR 0.71 and MMAE AUC GMR 1.90) (Table 2) [36]. The altered PK in brentuximab vedotin results in label recommendation to avoid use in patients with severe renal impairment [5].

The approach to evaluate organ dysfunction for ADC drug development remains situation dependent, but is trending toward a modeling and simulation approach. The population PK approach is routinely conducted to evaluate the impact of organ dysfunction on the exposure of ADC and its relevant analytes. If there is an impact on the exposure, such an impact on dose recommendation should be assessed in the context of benefit risk assessment and/or exposure-response relationship. In the future, physiologically based pharmacokinetic (PBPK) modeling approach may be used to assess the impact of organ dysfunction on ADC PK once the ADC 
PBPK model and organ dysfunction patient population is fully established.

\section{Drug-drug interactions}

Assessing drug-drug interaction (DDI) risk associated with ADCs needs to consider both the large- and smallmolecule components of the ADC. The cytotoxic payloads, upon release from ADCs, are expected to behave like small molecules and thus may be of concern for enzyme or transporter-mediated DDIs. The FDA and European Medicines Agency (EMA) have issued comprehensive recommendations for in vitro and in vivo studies to evaluate DDI potential for small molecules, but specific guidelines on DDI risk assessment for ADCs have not been issued. Given the relatively high potency and low systemic exposure of cytotoxic payloads, some unique DDI consideration might be needed for ADCs. Different from other molecules, human mass balance study is usually not conducted for most of the approved ADCs (6 out of 7 approved ADCs). Brentuximab vedotin is the only ADC that conducted a clinical excretion study but without complete recovery [21]. Instead, leveraging preclinical ADME data is the main strategy for initial DDI assessment of ADCs.

DDIs related to the payload have been extensively evaluated during the clinical development of an ADC. Table 3 summarizes the approaches, key findings and its implication on the drug label of payload-mediated DDIs for the seven approved ADCs, which include four different payloads: calicheamicin, MMAE, DM1, and DXd. Multiple approaches, namely dedicated clinical DDI study, theoretical risk assessment, physiologically based pharmacokinetic (PBPK) model, concomitant medication analysis, and referencing existing DDI data from a previously established ADC were used for DDI risk assessment. Theoretical risk assessment based on the in vitro DDI and clinical data is the most commonly used approach for the 7 ADCs (Table 3). Dedicated clinical DDI studies were conducted for two out of the seven ADCs: brentuximab vedotin and trastuzumab deruxtecan. PBPK modeling approach by leveraging available clinical DDI data for the same payload was used to inform DDI risk for polatuzumab vedotin, while exploratory concomitant medications analysis using NCA or population PK of clinical data to evaluate the effect of concomitant medications on payload PK was used for T-DM1.

Given low systemic concentrations of released payloads relative to its in vitro $\mathrm{K}_{\mathrm{i}} / \mathrm{IC}_{50}$ values of metabolizing enzymes and/or transporters, the risk for a payload to be a perpetrator of metabolizing enzymes and/or transporters is considered to be low. As shown in Table 3, most of these assessments are based on the theoretical risk assessments using the in vitro DDI and clinical data, which often results in the labeling statement such as, "at clinical relevant concentrations, the payload has no or low potential to inhibit the CYP enzymes and/or transporters". In vitro studies showed that MMAE and DM1 exhibited time-dependent and/or competitive inhibition of CYP3A with $\mathrm{K}_{\mathrm{i}}$ values in the micromolar range, however, the systemic levels of MMAE and DM1 released after administration of brentuximab vedotin and T-DM1 at their clinically approved doses are only in the nanomolar range [22, 23]. Consistent with these observations, a dedicated clinical DDI study showed that co-administration of brentuximab vedotin did not affect exposure to midazolam, a sensitive CYP3A substrate [38]. PBPK modeling by integrating the in vitro DDI and clinical data further confirms the low risk of MMAE for being a perpetrator for CYP3A substrates. The prediction results were highlighted in polatuzumab vedotin prescribing information [10].

In contrast, the potential for a released payload to be a DDI victim still exists, which could possibly impact safety as these payloads are highly potent and typically have a narrow or even no therapeutic window. As shown in Table 3, three out of the four payloads for the approved ADCs are metabolized by CYP3A with the exception of calicheamicin. In the case of calicheamicin, it has been shown that $\mathrm{N}$-acetyl gamma calicheamicin dimethyl hydrazide, the main circulating catabolite, is extensively metabolized, primarily via non-enzymatic reduction of the disulfide moiety, but not CYP enzymes, thus DDI risk for $N$-acetyl gamma calicheamicin dimethyl hydrazide as a victim of metabolizing enzymes is considered low and no additional assessment was conducted. Dedicated clinical studies were conducted for brentuximab vedotin and trastuzumab deruxtecan to assess the DDI risk for the released payload as a victim. Low magnitude of DDI interaction for MMAE and DXd was observed when co-administration with strong CYP3A inhibitors and inducers. Co-administration of trastuzumab deruxtecan with itraconazole (a strong CYP3A inhibitor) and ritonavir (a dual inhibitor of OATP1B/CYP3A) resulted in an $18 \%$ and $22 \%$, respectively, increase in steady-state exposure of DXd [25]. The magnitude of these changes is not considered clinically meaningful. In the case of brentuximab vedotin, co-administration with ketoconazole, strong CYP3A inhibitor, and rifampin, strong CYP3A inducer, increased MMAE exposure by $34 \%$ and decreased MMAE exposure by $\sim 46 \%$, respectively [38]. As increased exposure to MMAE may increase the risk of adverse reaction, close monitoring of adverse reactions is recommended when brentuximab vedotin is given concomitantly with strong CYP3A inhibitors [5]. Instead of conducting a clinical DDI study, polatuzumab vedotin, an MMAE-containing ADC with the same linker and payload as brentuximab vedotin, adopted a PBPK approach to project the magnitude of DDI with strong CYP3A inhibitors and inducers. The PBPK model 


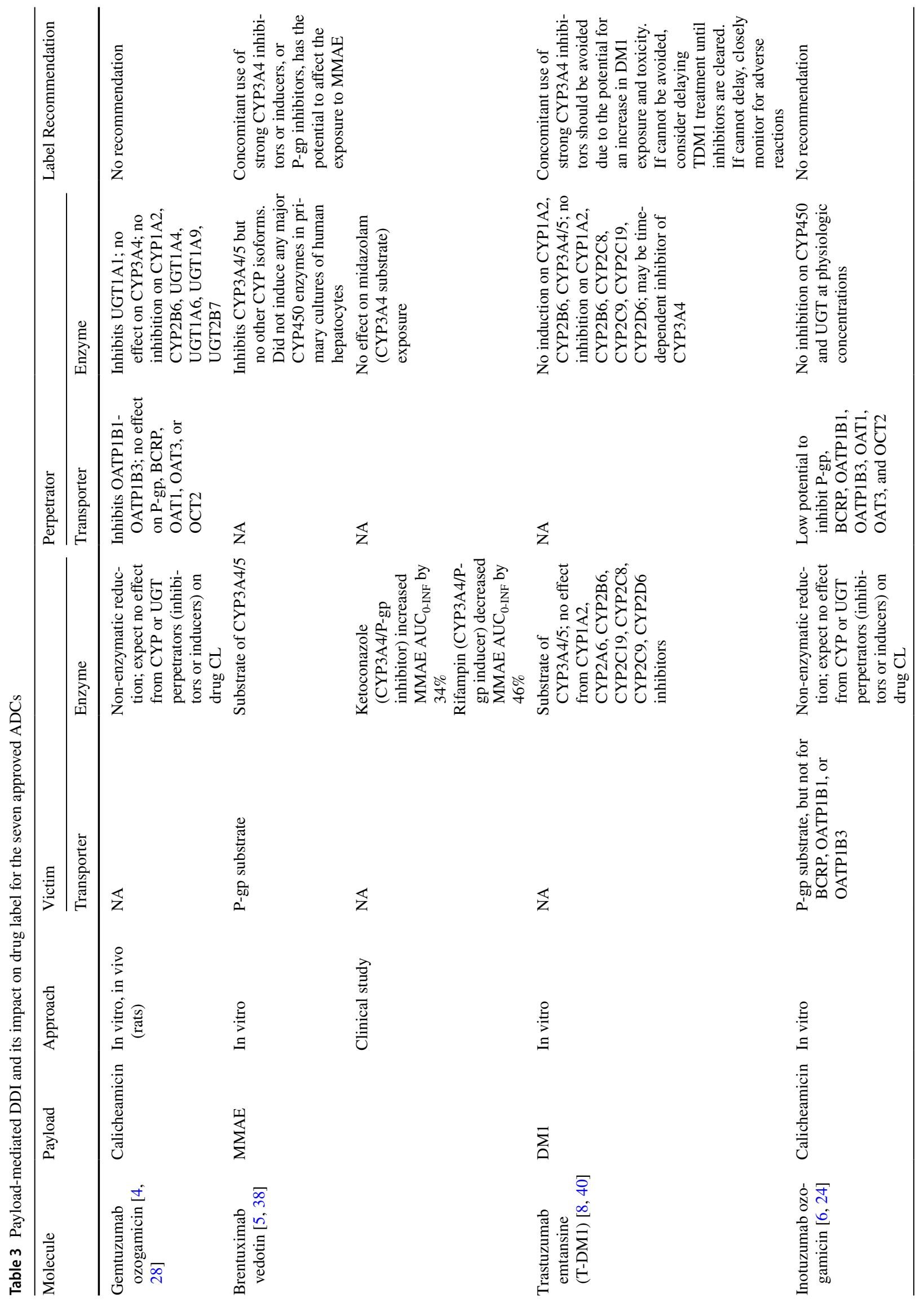




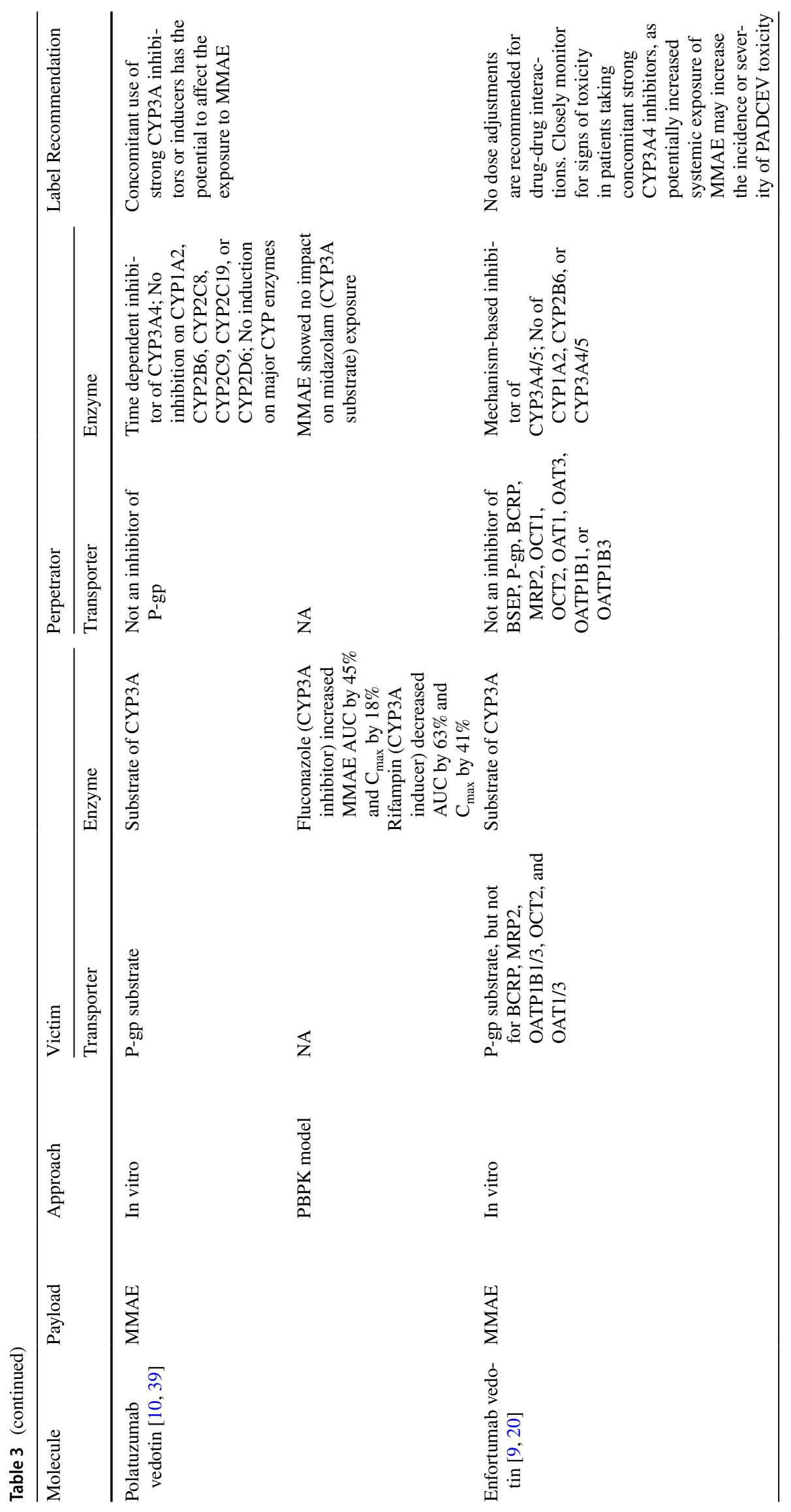




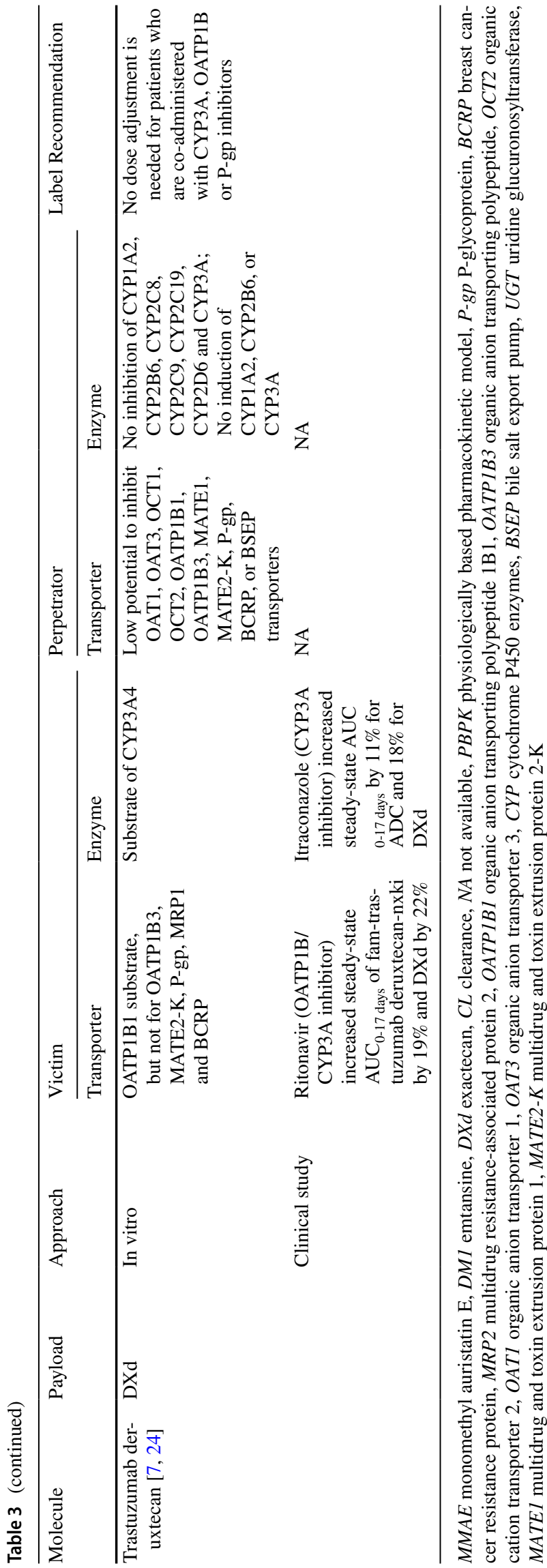

was developed using in silico and in vitro data and in vivo ADME and pharmacokinetic data of MMAE and a vcMMAE ADC and subsequently verified by the clinical DDI data of brentuximab vedotin [39]. The model projections were used to inform polatuzumab vedotin prescribing information. A slightly different approach was used for enfortumab vedotin, another MMAE-containing ADC, where its prescribing information simply refers to the clinical DDI results for brentuximab vedotin. For T-DM1, concomitant medication analysis with the Phase III pivotal clinical study showed that co-medication of CYP3A inhibitors and inducers does not result in any noticeable change in the pharmacokinetics of T-DM1 and DM1 [40]. However, given a dedicated clinical study was not conducted, caution language with detailed instructions was included in the T-DM1 label.

Assessing DDI risk associated with the mAb component of ADCs is often relatively rare since DDI involving mAbs are typically limited. Population PK approach is commonly used for such a DDI assessment. Population PK analysis of inotuzumab ozogamicin identified concomitant rituximab treatment as one of the significant covariates on inotuzumab ozogamicin clearance (CL decreased by $16 \%$ ). Similarly, population PK analysis with polatuzumab vedotin, which is approved for the treatment of $r / r$ DLBCL in combination with rituximab and bendamustine, showed that combination with rituximab had 24\% higher acMMAE exposure (ie, AUC) and 37-40\% lower exposure of unconjugated MMAE compared to patients receiving single-agent [26]. There was no apparent impact of bendamustine on polatuzumab vedotin and MMAE PK. It is worth noting that the magnitude of DDIs seen with concomitant medications was small and was not considered clinically relevant. Therefore, no dose adjustment is recommended for inotuzumab ozogamicin or polatuzumab vedotin in concomitant treatment with rituximab.

In summary, given the complex structure and unique PK characteristics of an ADC, risk-based DDI strategy by integrating both large and small-molecule components of an ADC is warranted to support the clinical development and approval of an ADC. Theoretical risk assessment using in vitro DDI and clinical data should be conducted based on FDA and EMA DDI guidelines. Depending on the level of risk, different approaches may be implemented to further assess the DDI potential. Modeling based approaches such as population PK and PBPK modeling, have become increasingly accepted and used to support DDI assessment and regulatory submission for ADCs.

\section{QTc assessment}

According to the ICH E14 [41] guidelines, it is generally recommended to evaluate the potential of non-antiarrhythmic drugs, such as ADCs to prolong the QT/QTc intervals 
in clinical development. A thorough QT study for an ADC is usually not feasible due to safety concerns on cytotoxicity of released payloads in healthy subjects and ethical concerns regarding a placebo arm in cancer patients. As an alternative, a clinical study that incorporates many of the key components of the thorough QT study is usually needed for an ADC, especially when there is evidence suggesting that the small-molecule component of the ADC or its catabolites are present in human systemic circulation.

Table 4 summarizes the approaches and results of QT assessment for the seven approved ADCs. In general, these seven ADCs did not show clinically meaningful impact on QTc prolongation, which is somewhat expected as the $\mathrm{mAb}$ component of the ADC is unlikely to interact with the human Ether-à-go-go-Related Gene (hERG) channel and the low concentrations of circulating payload after ADC dosing is unlikely to inhibit hERG channels in vivo. A dedicated clinical QT study was conducted for four out of the seven ADCs. The study design for brentuximab vedotin, T-DM1, and trastuzumab deruxtecan are similar, which involved a dedicated QT study collecting triplicate 12-lead ECG data with time-matched PK samples in $\sim 50$ cancer patients at a single dose level (i.e., clinical approved dose for brentuximab vedotin and T-DM1; a dose higher than clinical approved dose for trastuzumab deruxtecan). Gemtuzumab ozogamicin dedicated QT study is still ongoing ( $n=56$, NCT03727750). In comparison, inotuzumab ozogamicin, polatuzumab vedotin and enfortumab vedotin adopted a slightly different approach, instead of conducting a dedicated QT study, high-quality triplicate 12-lead ECG and time-matched PK samples were integrated in existing clinical Phase I and/or Phase II studies. Data pooled from one or multiple studies with $\sim 17-250$ cancer patients were used for QT assessment. It was noted that the majority of the approved ADCs had QT evaluation during cycles 1 and 3 representative of first dose and steady-state kinetics, except for enfortumab vedotin. Due to enfortumab vedotin's short half-life (3.4 days for ADC; 2.4 days for MMAE) and dosing on Days 1, 8 and 15 of a 28-day cycle (see Table 4), triplicate 12-lead ECGs were collected on days 1 and 3 and days 15 and 17 during the first 28-days cycle to capture the QTc effects at first dose and steady-state kinetics, respectively. Regardless of the study approaches, analysis of ECG data from clinical studies typically follows the ICH E14 [41] guidelines. For the seven approved ADCs, QT intervals corrected for heart rate using Frederica's formula (QTcF) are commonly used in concentration-QTc analysis. Three analytes (i.e., ADC conjugate, total antibody and unconjugated payload) were included in the concentration-QTc analysis for T-DM1, inotuzumab ozogamicin, polatuzumab vedotin while two analytes (i.e., ADC conjugate and unconjugated payload) used for brentuximab vedotin, enfortumab vedotin, trastuzumab deruxtecan (Table 4).
Overall, QTc risk for ADCs is expected to be low given the mAb component of the ADC and low levels of circulating payloads. Leveraging preclinical and clinical data such as in vitro hERG test, cardiac safety data in animals and the level of circulating payload, is important for developing appropriate ECG strategy in clinical studies. Additionally, ECG monitoring may not be warranted for ADCs with the circulating concentrations of the released payload similar or lower than those established as having no QT effect. Although dedicated QT studies have been conducted for the 4 approved ADCs, increasing trends showed that integrating high-quality ECG monitoring and exposure-QTc analysis to the existing phase I and/or II studies could be an effective way to assess overall risk and meet regulatory submission requirements.

\section{Exposure-response (ER) modeling}

Given a relatively narrow therapeutic window of ADCs [13] compared to mAbs, exposure-response (ER) analysis plays a critical role for supporting Phase II/III dose selection, label dose justification and guidance of dose adjustment for ADCs. Gemtuzumab ozogamicin dose is one of the examples highlighting the importance of ER analysis for selecting appropriate dose and schedule. Gemtuzumab ozogamicin was first granted an accelerated approval in 2000 as a monotherapy with dose of $9 \mathrm{mg} / \mathrm{m}^{2}$ for the treatment of patients with CD33 positive acute myeloid leukemia, however, the sponsor withdrew gemtuzumab ozogamicin from the market in 2011 as the confirmative study failed to demonstrate better efficacy but showed higher rates of fatal hepatotoxicity and veno-occlusive disease (VOD). Exploratory ER analyses of gemtuzumab ozogamicin using data from single agent of $9 \mathrm{mg} / \mathrm{m}^{2}$ dose showed that the risk for VOD increases as Cmax after first dose of gemtuzumab ozogamicin increases, while exposure-efficacy (i.e., complete remission) relationship, however, was relatively flat for any exposure measure including $C_{\max }$ after first dose, indicating a fractionated lower dose may have the potential to reduce the risk for VOD but preserve the efficacy of gemtuzumab ozogamicin. Recent positive study read-out with fractionated dosing of $3 \mathrm{mg} / \mathrm{m}^{2}$ confirmed the above hypothesis and demonstrated improved clinical benefit with reduced VOD risk, thus leading to the re-approval of gemtuzumab ozogamicin in 2016 [42, 43].

One of unique features of ADC ER analysis which is different from other therapies, is that it requires comprehensive understanding which analyte(s) are the key drive for efficacy and safety due to the complex structure of ADCs. Based on the mechanism of action, ADC conjugate, measured as conjugated antibody or conjugated payload, is generally believed to be the key analyte of interest to drive safety and efficacy for an ADC. However, it is worth noting that 


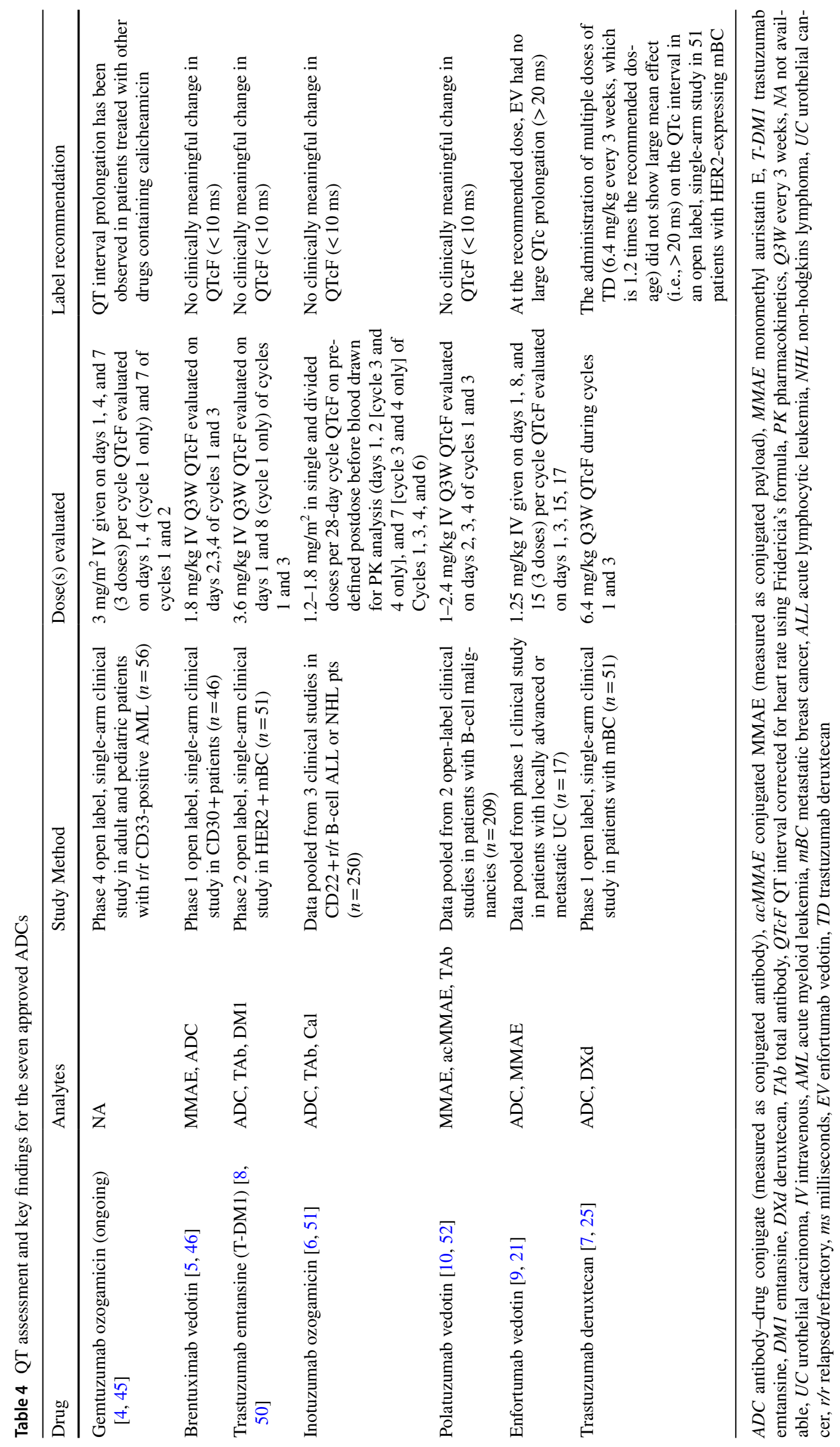


released payloads are highly potent and may possibly pose a safety risk, exposures of unconjugated drug are sometimes included in the exposure-safety analysis. Table 5 summarizes the ER results for the seven approved ADC. Among the seven approved ADCs, four of the ADCs, namely brentuximab vedotin, polatuzumab vedotin, enfortumab vedotin and trastuzumab deruxtecan, included both ADC conjugate and unconjugated drug analytes in their ER analyses. A positive exposure-efficacy relationship with ADC conjugate exposure was consistently observed for the four ADCs, however, no apparent or negative exposure-efficacy relationship was observed for unconjugated drug exposure. In comparison, exposure-safety relationships for the four ADCs vary, depending on safety endpoints and analytes used in the analyses. For brentuximab vedotin and enfortumab vedotin, ADC conjugate exposure appeared to correlate better with safety than that of unconjugated drug. In the case of brentuximab vedotin, a positive exposure-safety relationship was observed with ADC conjugate exposure, but not with that of unconjugated drug, while for enfortumab vedotin, positive exposure-safety relationships were observed with exposure of both ADC conjugate and unconjugated drug, but the strengthen of exposure-safety relationship appears to be much weaker for unconjugated drug. For polatuzumab vedotin and trastuzumab deruxtecan, no consistent exposure-safety trends were observed; positive exposure-safety relationships were observed sparsely between some safety endpoints and exposure of ADC conjugate and/or unconjugated payload. For T-DM1, inotuzumab ozogamicin and gemtuzumab ozogamicin, only one analyte was used in their ER analyses. Specifically, ADC conjugate was used for ER analyses of T-DM1 and inotuzumab ozogamicin. For both ADCs, increased conjugate exposure appeared to be associated with improved efficacy (i.e., ORR, PFS, OS). No apparent positive exposure-safety relationship was observed with T-DM1 treatment (i.e., hepatotoxicity and thrombocytopenia), while a positive exposure-efficacy relationship was founded between inotuzumab ozogamicin and some of treatment-related AE (i.e., Grade 3 + thrombocytopenia and HEAB-assessed VOD). Given ADC conjugate was not measured for gemtuzumab ozogamicin, total antibody analyte was used for the ER analysis instead. Together, for most of the seven approved ADCs, the efficacy endpoints appear to correlate best with ADC conjugate compared to that of unconjugated payload. For safety outcomes, while ADC exposures were often correlated with AEs, unconjugated payload exposures may also be important for certain AEs. Total antibody analyte was usually not included in the ER analysis since there is a high correlation between conjugate and the total antibody exposures [44].

It is worth noting that four out of the seven ADCs (i.e., gemtuzumab ozogamicin, brentuximab vedotin, T-DM1 and enfortumab vedotin) use the data from single dose level in the exposure-efficacy analysis given efficacy data is indication-specific and only one dose level is usually studied in the pivotal study. Similar to ER analysis of other cancertargeting biologics, caution needs to be taken to interpret the ER results of an ADC when the analyses are performed with data with only single dose levels as the effect of disease severity on ADC exposure may confound ER relationship (i.e., a visual steep trend is seen when the true relationship is flat) $[45,46]$. The exposure-safety, however, is less likely to be confounded as the safety data are often pooled across the multiple studies, dose levels and patient populations. As illustrated in Table 5, a range of clinically tested doses were included in the ER safety analysis for most of the seven approved ADCs, while only three out of the seven ADCs include multiple dose levels in the ER efficacy analyses.

In summary, ER analysis provided valuable information beyond dose confirmation of the clinically tested dosage regimen in the phase 3 studies. We have illustrated the impact of ER analyses of gemtuzumab ozogamicin to enable test a fractionated lower dose thus leading to the re-approval of gemtuzumab ozogamicin. Additionally, ER analyses could guide dose adjustment. For brentuximab vedotin, the positive ER relationships with peripheral neuropathy and neutropenia support the dose reduction recommendation from 1.8 to $1.2 \mathrm{mg} / \mathrm{kg}$ in the event of Grade $2+$ peripheral neuropathy and Grade $4+$ neutropenia [22]. Furthermore, ER analysis could be used to identify the appropriate therapeutic dose for phase 2. For trastuzumab deruxtecan, ER analysis identified two potential phase 2 doses of 5.4 and $6.4 \mathrm{mg} / \mathrm{kg}$ from phase 1 data and confirmed the final dose recommendation of $5.4 \mathrm{mg} / \mathrm{kg}$ in pivotal studies based on similar predicted ORR probability (ORR 90\% CI $0.63[0.55,0.70]$ and 0.68 $[0.58,0.77]$ for $5.4 \mathrm{mg} / \mathrm{kg}$ and $6.4 \mathrm{mg} / \mathrm{kg}$, respectively) and exposure-safety relationships with greater rate of AEs in the $6.4 \mathrm{mg} / \mathrm{kg}$ group compared to the $5.4 \mathrm{mg} / \mathrm{kg}$ group [25].

\section{Summary and future directions}

ADCs represent a rapidly evolving area of oncology drug development and hold significant promise. The complex structure of ADCs poses unique challenges to clinical pharmacology strategy in supporting development and approval of ADCs, since it requires a quantitative understanding of the PK and PD properties of multiple different molecular species (e.g., ADC conjugate, total antibody and unconjugated payload) in the systemic circulation and/or tissues of interest (e.g., tumors). Integration of diverse clinical pharmacology approaches, ranging from dedicated clinical pharmacology studies (e.g., DDI, QTc, renal/hepatic impairment study) to mechanistic and/or empirical models (e.g., PBPK, population PK modeling for one- or two- analytes, exposure-response analysis) can provide insights into the PK, PD 
760

Cancer Chemotherapy and Pharmacology (2021) 87:743-765

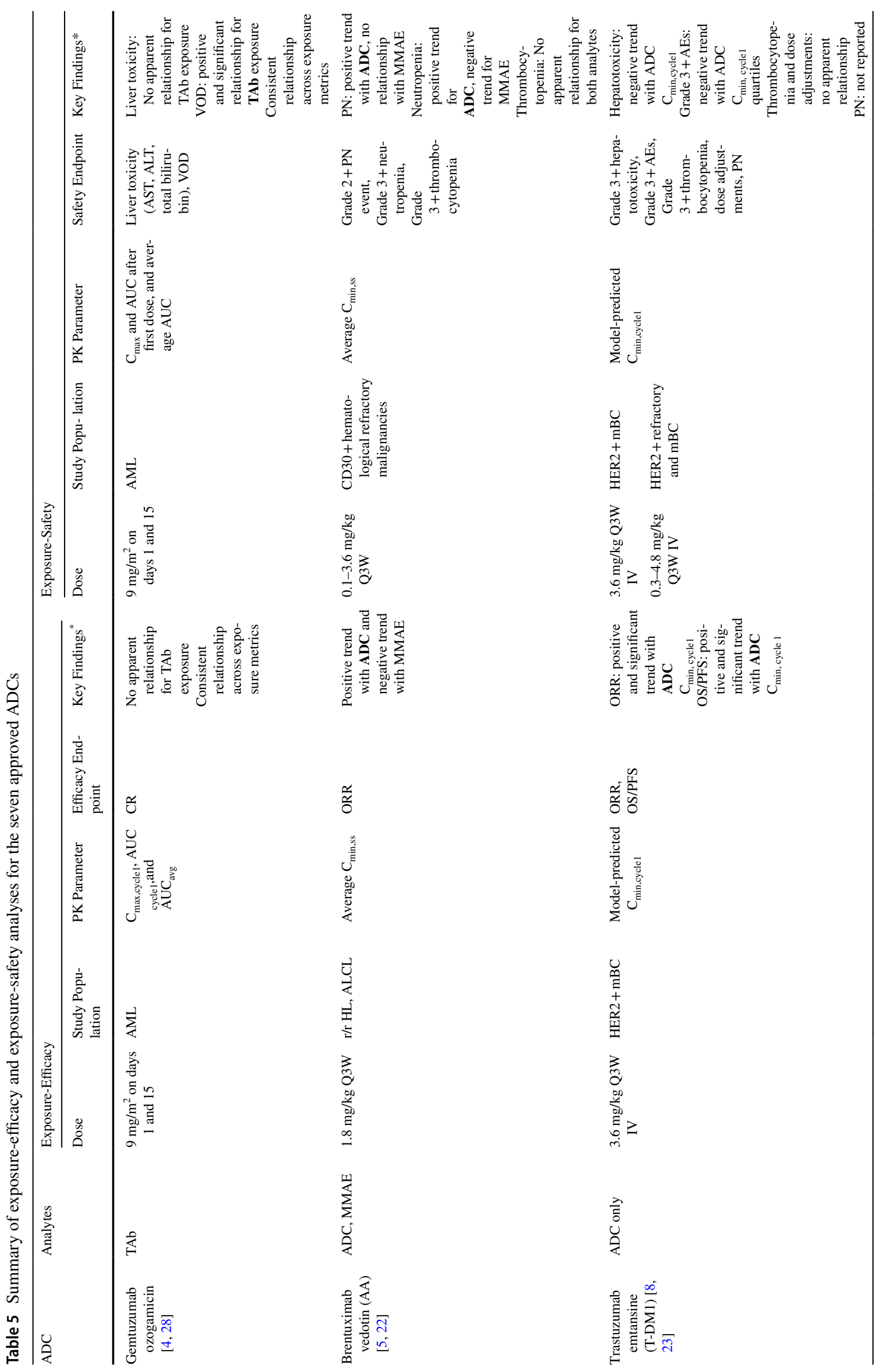

Springer 


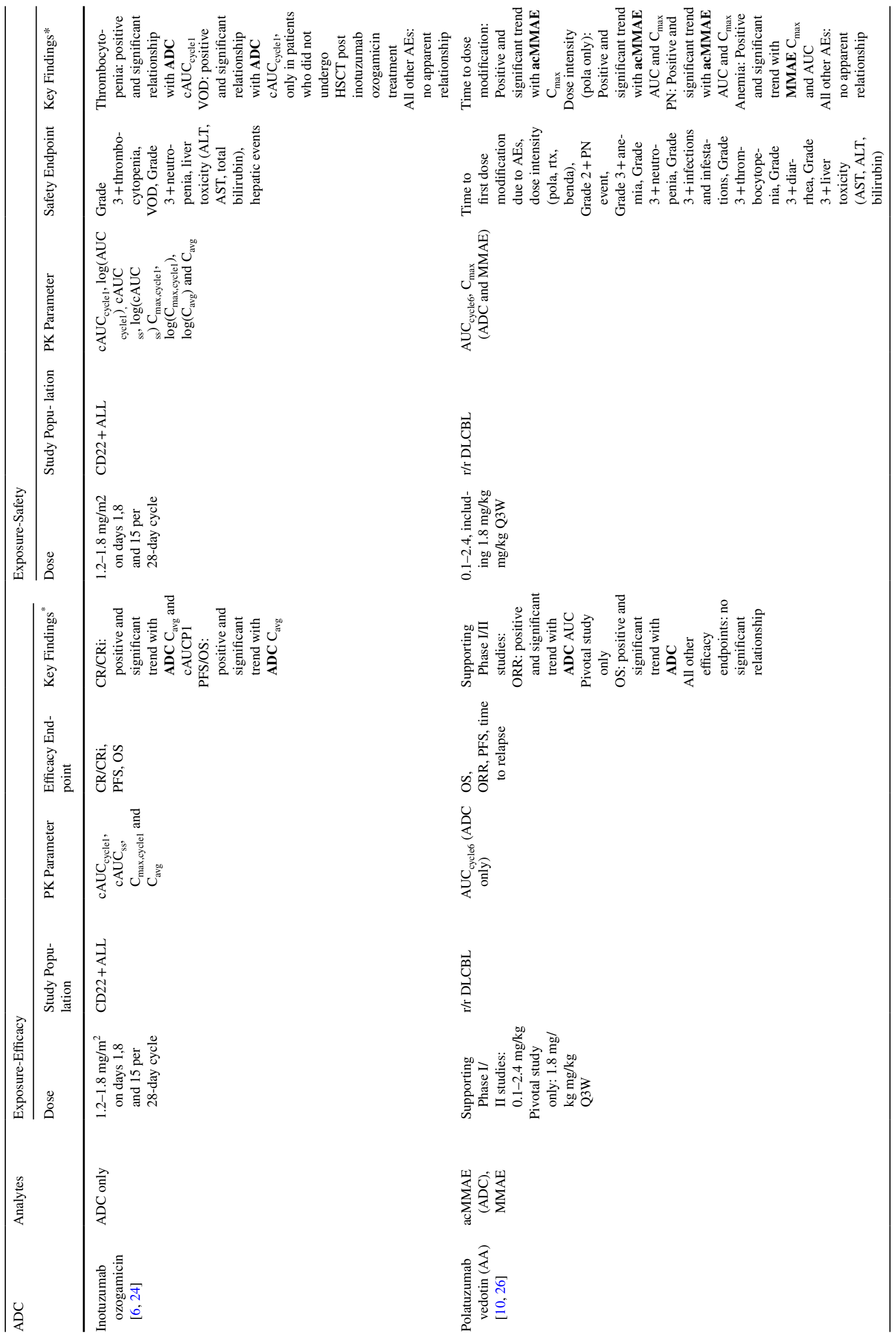




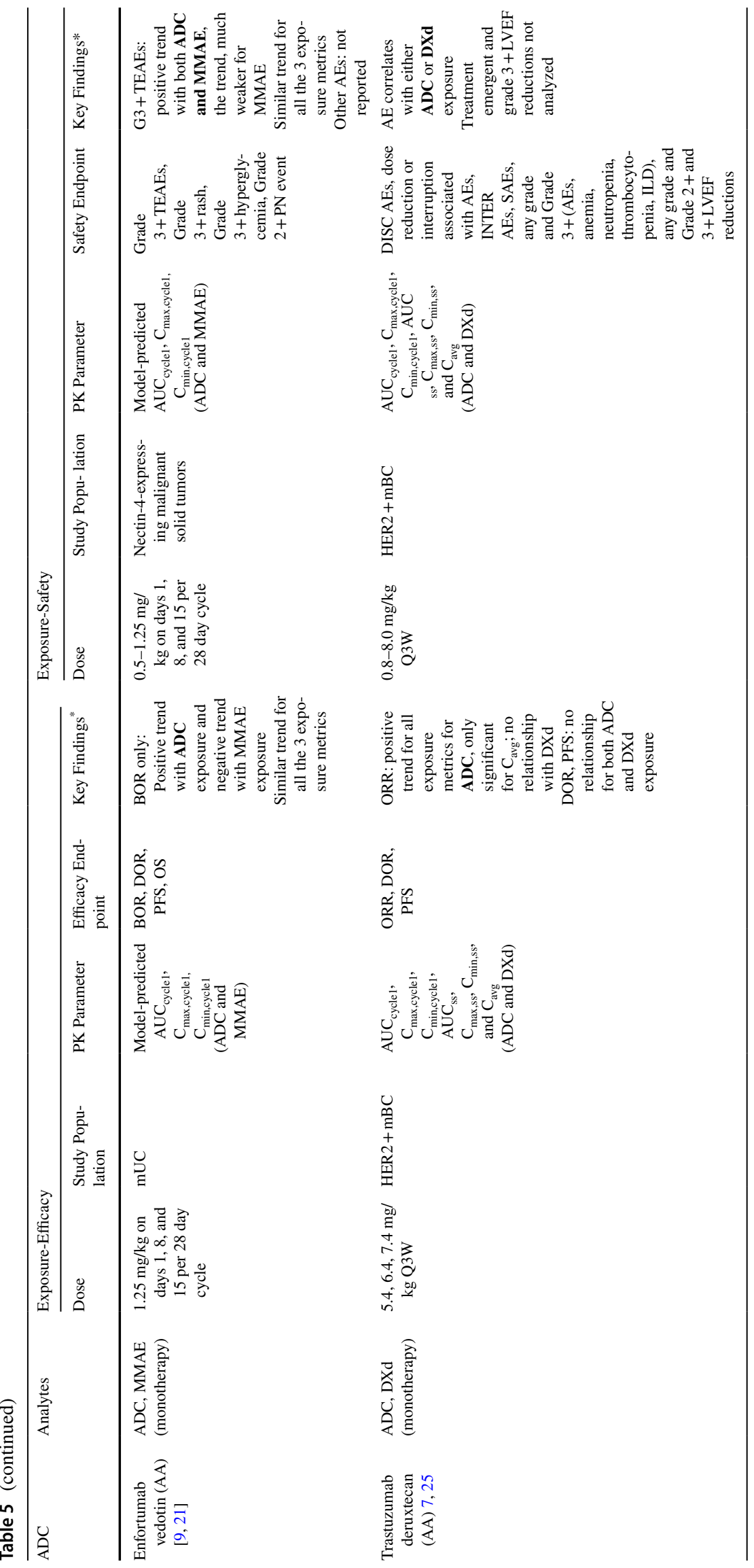

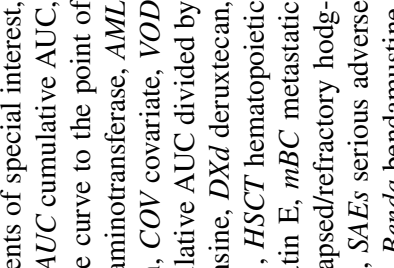
ठ․․

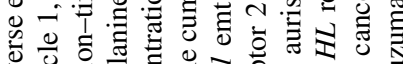

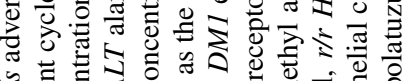

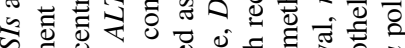

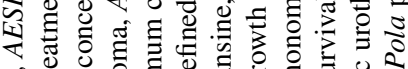

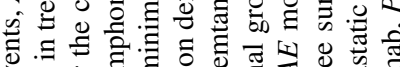

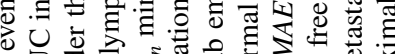

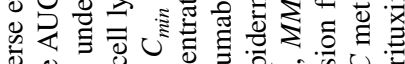

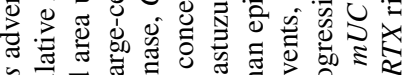

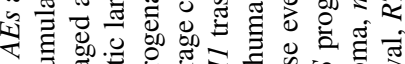

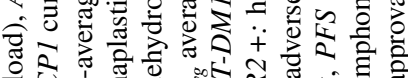
टे

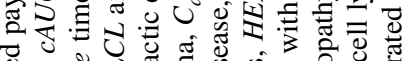

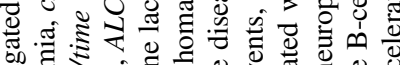

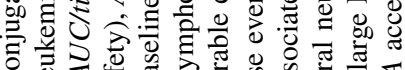
U.

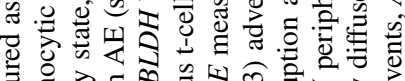

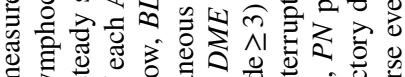

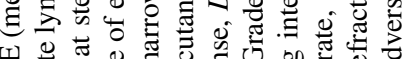
少号

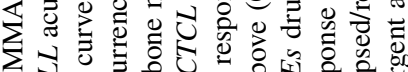

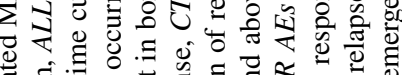

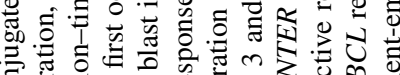

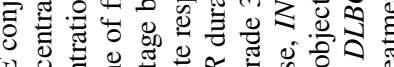

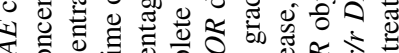
o ठै

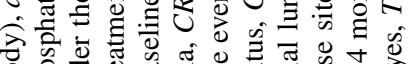

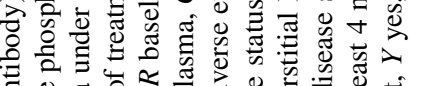

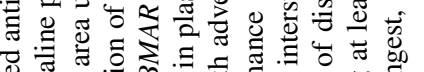

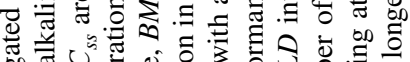

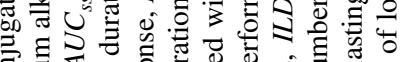

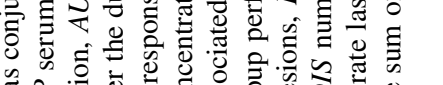

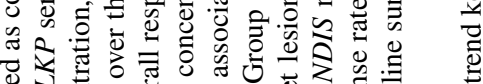

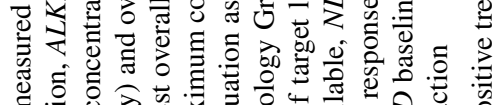

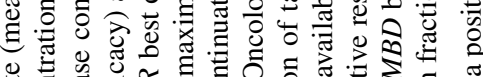

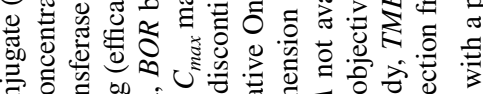

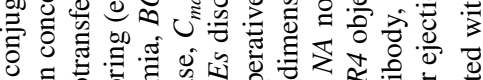

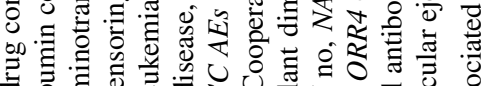

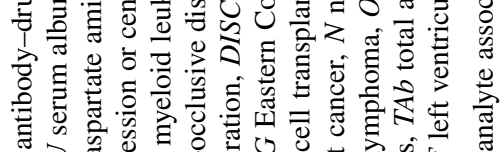

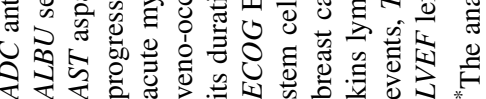


and ADME properties of an ADC and inform development decision and clinical dose and schedule selection (Fig. 1). An additional consideration for clinical development not discussed in this review includes the thorough assessment of immunogenicity on ADC PK, efficacy, and safety.

As the field continues to evolve, the selection of suitable ADC targets and the identification of a target population remain critical challenges. Efforts to further optimize "next-generation" ADCs using engineered antibodies, innovative linkers, conjugation methods, and novel payloads are rapidly advancing. Despite the great success of ADCs, it is worth noting that the therapeutic window for ADCs remains relatively narrow with the maximum tolerated dose (MTD) often reached before ADCs achieve the maximum efficacious dose. Additionally, the toxicities associated with the ADCs might dictate the number of treatment cycles that the patients can tolerate and often result in dose delay, dose reductions or study discontinuation [13]. The future success of ADCs in part will depend on our ability to overcome these developmental challenges, especially by developing clear strategies to optimize the dose and schedule of ADCs and identifying predictive biomarkers to assess response, optimize patient selection, and inform potential combination therapies.

Open Access This article is licensed under a Creative Commons Attribution 4.0 International License, which permits use, sharing, adaptation, distribution and reproduction in any medium or format, as long as you give appropriate credit to the original author(s) and the source, provide a link to the Creative Commons licence, and indicate if changes were made. The images or other third party material in this article are included in the article's Creative Commons licence, unless indicated otherwise in a credit line to the material. If material is not included in the article's Creative Commons licence and your intended use is not permitted by statutory regulation or exceeds the permitted use, you will need to obtain permission directly from the copyright holder. To view a copy of this licence, visit http://creativecommons.org/licenses/by/4.0/.

\section{References}

1. Boni V, Sharma MR, Patnaik A (2020) The resurgence of antibody drug conjugates in cancer therapeutics: novel targets and payloads. Am Soc Clin Oncol Educ Book 40:1-17

2. FDA. Drugs@FDA: FDA-approved drugs. https://www.acces sdata.fda.gov/scripts/cder/daf/

3. FDA. Press Release. https://www.fda.gov/news-events/pressannouncements/fda-approves-mylotarg-treatment-acute-myelo id-leukemia

4. Mylotarg (2000) (gemtuzumab ozogamicin) prescribing information (USPI); Philadelphia (PA): Pfizer Wyeth Pharmaceuticals, Inc.; Reference ID: 4147588. https://www.pfizerpro.com/produ ct/mylotarg

5. Adcetris (2011) (brentuximab vedotin) prescribing information (USPI); Bothell (WA): Seattle Genetics, Inc.; Reference ID:
3662552. https://www.accessdata.fda.gov/drugsatfda_docs/label/ 2014/125388_S056S078lbl.pdf

6. Besponsa (2011) (inotuzumab ozogamicin) prescribing information (USPI); Philadelphia (PA): Pfizer Wyeth Pharmaceuticals, Inc.; Reference ID: 4140675. https://www.accessdata.fda.gov/ drugsatfda_docs/label/2017/761040s000lbl.pdf

7. Enhertu (2019) (fam-trastuzumab deruxtecan-nxki) prescribing information (USPI); Basking Ridge (NJ): Daiichi Sankyo, Inc.; Reference ID: 4537820. https://www.accessdata.fda.gov/drugs atfda_docs/label/2019/761139s000lbl.pdf

8. Kadcyla (2013) (ado-trastuzumab emtansine) prescribing information (USPI); South San Francisco (CA): Genentech, Inc; Reference ID: 4428535. https://www.accessdata.fda.gov/drugsatfda docs/label/2019/125427s105lbl.pdf

9. Padcev (2019) (enfortumab vedotin-ejfv) prescribing information (USPI); Bothell (WA): Seattle Genetics, Inc.; Reference ID: 4536404. https://www.accessdata.fda.gov/drugsatfda_docs/label/ 2019/761137s000lbl.pdf

10. Polivy (2019) (polatuzumab vedotin-piiq) prescribing information (USPI);South San Francisco (CA): Genentech, Inc; https:// www.genentech-access.com/hcp/brands/polivy.html?c=gas-16a14 d7526a\&gclid=EAIaIQobChMIxpennImP6gIVZz6tBh0SHAc oEAAYASAAEgIbvfD_BwE\&gclsrc=aw.ds

11. Trodelvy (2020) Trodelvy (sacituzumab govitecan-hziy) prescribing information (USPI); Morris Plains (NJ): Immunomedics Inc.; Reference ID: 4596074. https://www.accessdata.fda.gov/drugs atfda_docs/label/2020/761115s000lbl.pdf

12. BLENREP (2020) (belantamab mafodotin-blmf) prescribing information (USPI); Middlesex (UK): Glaxosmithilne property development Ltd. England; https://www.accessdata.fda.gov/drugs atfda_docs/label/2020/761158s000lbl.pdf

13. Coats S, Williams M, Kebble B, Dixit R, Tseng L, Yao NS, Tice DA, Soria JC (2019) Antibody-drug conjugates: future directions in clinical and translational strategies to improve the therapeutic index. Clin Cancer Res 25:5441-5448

14. Kamath AV, Iyer S (2015) Preclinical pharmacokinetic considerations for the development of antibody drug conjugates. Pharm Res 32:3470-3479

15. Kamath AV, Iyer S (2016) Challenges and advances in the assessment of the disposition of antibody-drug conjugates. Biopharm Drug Dispos 37:66-74

16. Shen BQ, Xu K, Liu L, Raab H, Bhakta S, Kenrick M, ParsonsReponte KL, Tien J, Yu SF, Mai E et al (2012) Conjugation site modulates the in vivo stability and therapeutic activity of antibody-drug conjugates. Nat Biotechnol 30:184-189

17. Gorovits B, Alley SC, Bilic S, Booth B, Kaur S, Oldfield P, Purushothama S, Rao C, Shord S, Siguenza P (2013) Bioanalysis of antibody-drug conjugates: American Association of Pharmaceutical Scientists Antibody-Drug Conjugate Working Group position paper. Bioanalysis 5:997-1006

18. Lin K, Tibbitts J (2012) Pharmacokinetic considerations for antibody drug conjugates. Pharm Res 29:2354-2366

19. Kaur S, Xu K, Saad OM, Dere RC, Carrasco-Triguero M (2013) Bioanalytical assay strategies for the development of antibodydrug conjugate biotherapeutics. Bioanalysis 5:201-226

20. Saad OM, Shen BQ, Xu K, Khojasteh SC, Girish S, Kaur S (2015) Bioanalytical approaches for characterizing catabolism of antibody-drug conjugates. Bioanalysis 7:1583-1604

21. CDR. Center for Drug Evaluation and Research. MULTI-DISCIPLINE REVIEW of enfortumab vedotin, APPLICATION NUMBER:761137Orig1s000 https://www.accessdata.fda.gov/ drugsatfda_docs/nda/2019/7611370rig1s000MultiDiscliplineR. pdf

22. CDR. Center for Drug Evaluation and Research. Clinical Pharmacology and Biopharmaceutics Review for brentuximab vedotin, APPLICATION NUMBER: 125388Orig1s000. https://www.acces 
sdata.fda.gov/drugsatfda_docs/nda/2011/125388Orig1s000ClinP harmR.pdf

23. CDR. Center for Drug Evaluation and Research. MULTI-DISCIPLINE REVIEW of trastuzumab emtansine APPLICATION NUMBER:125427Orig1s000. https://www.accessdata.fda.gov/ drugsatfda_docs/nda/2013/125427Orig1s000ClinPharmR.pdf

24. CDR. Center for Drug Evaluation and Research. MULTI-DISCIPLINE REVIEW of inotuzumab ozogamicin, APPLICATION NUMBER:761040Orig1s000. https://www.accessdata.fda.gov/ drugsatfda_docs/nda/2017/761040Orig1s000MultidisciplineR.pdf

25. CDR. Center for Drug Evaluation and Research. MULTI-DISCIPLINE REVIEW of trastuzumab deruxtecan, APPLICATION NUMBER:761139Orig1s000. https://www.accessdata.fda.gov/ drugsatfda_docs/nda/2019/761139Orig1s000MultidisciplineR.pdf

26. CDR. Center for Drug Evaluation and Research. MULTI-DISCIPLINE REVIEW of polatuzumab vedotin, APPLICATION NUMBER:7611210rig1s000. https://www.accessdata.fda.gov/ drugsatfda_docs/nda/2019/761121Orig1s000ClinPharmR.pdf

27. Dowell JA, Korth-Bradley J, Liu H, King SP, Berger MS (2001) Pharmacokinetics of gemtuzumab ozogamicin, an antibody-targeted chemotherapy agent for the treatment of patients with acute myeloid leukemia in first relapse. J Clin Pharmacol 41:1206-1214

28. CDR. Center for Drug Evaluation and Research. MULTI-DISCIPLINE REVIEW of gemtuzumab ozogamicin APPLICATION NUMBER:761060Orig1s000O 761060Orig2s000. https://www. accessdata.fda.gov/drugsatfda_docs/nda/2017/761060Orig1s000 Orig1Orig2s000SumR.pdf

29. Li H, Han TH, Hunder NN, Jang G, Zhao B (2017) Population pharmacokinetics of Brentuximab Vedotin in patients with CD30-expressing hematologic malignancies. J Clin Pharmacol 57:1148-1158

30. Lu D, Lu T, Gibiansky L, Li X, Li C, Agarwal P, Shemesh CS, Shi $\mathrm{R}$, Dere RC, Hirata J et al (2020) Integrated two-analyte population pharmacokinetic model of Polatuzumab Vedotin in patients with non-hodgkin lymphoma. CPT Pharmacometrics Syst Pharmacol 9:48-59

31. Lu D, Girish S, Gao Y, Wang B, Yi JH, Guardino E, Samant M, Cobleigh M, Rimawi M, Conte P et al (2014) Population pharmacokinetics of trastuzumab emtansine (T-DM1), a HER2-targeted antibody-drug conjugate, in patients with HER2-positive metastatic breast cancer: clinical implications of the effect of covariates. Cancer Chemother Pharmacol 74:399-410

32. Hibma J, Knight B (2019) Population pharmacokinetic modeling of Gemtuzumab Ozogamicin in adult patients with acute myeloid leukemia. Clin Pharmacokinet 58:335-347

33. Lucas AT, Robinson R, Schorzman AN, Piscitelli JA, Razo JF, Zamboni WC (2019) Pharmacologic considerations in the disposition of antibodies and antibody-drug conjugates in preclinical models and in patients. Antibodies (Basel) 8(1):3

34. Hedrich WD, Fandy TE, Ashour HM, Wang H, Hassan HE (2018) Antibody-drug conjugates: pharmacokinetic/pharmacodynamic modeling, preclinical characterization, clinical studies, and lessons learned. Clin Pharmacokinet 57(6):687-703

35. Sun Q, Seo S, Zvada S, Liu C, Reynolds K (2020) Does hepatic impairment affect the exposure of monoclonal antibodies? Clin Pharmacol Ther 107:1256-1262

36. Zhao B, Chen R, O'Connor OA, Gopal AK, Ramchandren R, Goy A, Matous JV, Fasanmade AA, Manley TJ, Han TH (2016) Brentuximab vedotin, an antibody-drug conjugate, in patients with CD30-positive haematologic malignancies and hepatic or renal impairment. Br J Clin Pharmacol 82:696-705

37. Li C, Agarwal P, Gibiansky E, Jin JY, Dent S, Goncalves A, Nijem I, Strasak A, Harle-Yge ML, Chernyukhin N et al (2017) A Phase I Pharmacokinetic Study of Trastuzumab Emtansine (T-DM1) in patients with human epidermal growth factor receptor 2-positive metastatic breast cancer and normal or reduced hepatic function. Clin Pharmacokinet 56:1069-1080

38. Han TH, Gopal AK, Ramchandren R, Goy A, Chen R, Matous JV, Cooper M, Grove LE, Alley SC, Lynch CM et al (2013) CYP3A-mediated drug-drug interaction potential and excretion of brentuximab vedotin, an antibody-drug conjugate, in patients with CD30-positive hematologic malignancies. J Clin Pharmacol 53:866-877

39. Chen Y, Samineni D, Mukadam S, Wong H, Shen BQ, Lu D, Girish S, Hop C, Jin JY, Li C (2015) Physiologically based pharmacokinetic modeling as a tool to predict drug interactions for antibody-drug conjugates. Clin Pharmacokinet 54:81-93

40. Agarwal P, Wang B, Li C, Lu M, Kang B, Chernyukhin S (2015) Assessment of the potential for drug-drug interactions between Trastuzumab Emtansine (T-DM1) and CYP3A in-hibitors or inducers and the impact on its PK and safety. ASCPT 2015. New Orleans, Louisiana

41. FDA. FDA (2005) International Conference on Harmonisation (ICH) - Guidance for Industry: E14 clinical evaluation of QT/ QTc interval prolongation and proarrhythmic potential for nonantiarrhythmic drugs. http://www.fda.gov/RegulatoryInformation/ Guidances/ucm129335.htm

42. Amadori S, Suciu S, Selleslag D, Aversa F, Gaidano G, Musso M, Annino L, Venditti A, Voso MT, Mazzone C et al (2016) Gemtuzumab ozogamicin versus best supportive care in older patients with newly diagnosed acute myeloid leukemia unsuitable for intensive chemotherapy: results of the randomized phase III EORTC-GIMEMA AML-19 trial. J Clin Oncol 34:972-979

43. Castaigne S, Pautas C, Terre C, Raffoux E, Bordessoule D, Bastie JN, Legrand O, Thomas X, Turlure P, Reman O et al (2012) Effect of gemtuzumab ozogamicin on survival of adult patients with denovo acute myeloid leukaemia (ALFA-0701): a randomised, openlabel, phase 3 study. Lancet 379:1508-1516

44. Li C, Zhang C, Deng R, Leipold D, Li D, Latifi B, Gao Y, Zhang C, Li Z, Miles D et al (2019) Prediction of human pharmacokinetics of antibody-drug conjugates from nonclinical data. Clin Transl Sci 12:534-544

45. Liu J, Wang YF, Zhao L (2015) Assessment of exposure-response $(\mathrm{E}-\mathrm{R})$ and case-control $(\mathrm{C}-\mathrm{C})$ analyses in oncology using simulation based approach. (Abstract W-34). American Conference of Pharmacometrics Annual Meeting

46. Wang Y (2016) Special considerations for modeling exposure response for biologics. American Society for Clinical Pharmacology and Therapeutics Annual Meeting

47. Garrett M, Ruiz-Garcia A, Parivar K, Hee B, Boni J (2019) Population pharmacokinetics of inotuzumab ozogamicin in relapsed/ refractory acute lymphoblastic leukemia and non-Hodgkin lymphoma. J Pharmacokinet Pharmacodyn 46:211-222

48. https://clinicaltrials.gov/ct2/show/results/NCT03727750

49. Han TH, Chen R, Advani R, Berryman RB, Smith SE, ForeroTorres A, Rosenblatt JD, Smith MR, Zain J, Hunder NN et al (2013) Brentuximab vedotin does not cause clinically relevant QTc interval prolongation in patients with CD30-positive hematologic malignancies. Cancer Chemother Pharmacol 72:241-249

50. Gupta M, Wang B, Carrothers TJ, LoRusso PM, Chu YW, Shih T, Loecke D, Joshi A, Saad O, Yi JH et al (2013) Effects of trastuzumab emtansine (T-DM1) on QT interval and safety of pertuzumab plus T-DM1 in patients with previously treated human epidermal growth factor receptor 2-positive metastatic breast cancer. Clin Pharmacol Drug Dev 2:11-24

51. Hibma JE, Kantarjian HM, DeAngelo DJ, Boni JP (2019) Effect of inotuzumab ozogamicin on the QT interval in patients with haematologic malignancies using QTc-concentration modelling. Br J Clin Pharmacol 85:590-600

52. Lu D, Girish S, Marchand M, Mouksassi M, Garnett C, Li C, Agarwal P, Essig J, Jones C, Hirata J, Chu, Jin J (2016) 
Concentration-QTC Analysis of Polatuzumab Vedotin in Patients with B-Cell Hematologic Malignancies (conference paper S5-S5). Conference of Clinical Pharmacology and Therapeutics Annual Meeting
Publisher's Note Springer Nature remains neutral with regard to jurisdictional claims in published maps and institutional affiliations. 